\title{
Influence of magnetized water and some fertilizers on the growth of two cultivars of (Pistacia vera $\mathrm{L}$.) seedling
}

\author{
Hardy Kakakhan Awlla Shekhany ${ }^{1}$ and Sawsan mohamed-saeed Ali \\ Kanimarani $^{2}$ \\ (Forestry and Horticulture dept. College of Agriculture/University of Salahaddin, Erbil, Kurdistan region, Iraq)
}

\begin{abstract}
The effects of magnetic water, NPK and B doses on some growth characteristics of two cultivars of (Pistacia vera L.) seedlings was studied. The following treatments were employed: 1-Kalle-Ghochi and Mussili cultivars; 2- magnetic water levels at 0, 155, 3000gauss; 3-NPK (20:20:20) at o, 250, 500 and 1000ppm; 4- B at 0,5 , and 10 and20ppm. The interaction among treatments had highly significant effects on most characteristics. Generally, effect of the two cultivars was varied, while magnetic water treatment was reducing root growth especially with NPK interaction when the highest values for root length, root wet and dry weights $(34.00 \mathrm{~cm}, 44.76 \mathrm{gm}$ and $36.07 \mathrm{gm}$ respectively) were recorded from untreated seedlings, while the interaction of magnetic water levels and NPK had promotive effects on the vegetative parameters. The interaction of $B$, cultivars and magnetic water levels had significant effects on studied parameters, the most effective dose of $B$ was 20ppm. The interaction among the four studied factors had variable results, the best values as follows: Musilli seedlings irrigated with tap water treated with 500ppm NPK gave the highest values of leaf area and shoot length in different Boron doses (20 and 10 ppm respectively). The highest number of leaves (51.33) was recorded from Kalle-Ghochi seedlings treated with 1500 Gauss magnetic water, 1000ppm NPK and 10ppm Boron. The thickest diameter of seedlings $(8.36 \mathrm{~mm})$ was measured from Kalle-Ghochi cultivar treating with 3000 Gauss magnetic water and 10ppm Boron. The highest shoot wet and dry weights (59.26gm and 44.13gm respectively) were resulted from Kalle-Ghochi seedlings irrigated with tap water treated with 1000ppm NPK and 20ppm Boron, and the longest root $(41.50 \mathrm{~cm})$ was recorded with the same above treatment but with no NPK doses. the best chlorophyll content (60.85\%) resulted in Kalle-Ghochi seedlings treated with 1500 Gauss magnetic water, 250ppm NPK and 10ppm Boron, while the best RWC (10.70\%) was measured from Musilli seedlings irrigated with 3000 Gauss magnetic water, 1000ppm NPK and 5ppm Boron.
\end{abstract}

Keywords: (Pistacia vera L.) cultivars, magnetic water, NPK, B, growth parameter.

\section{Introduction}

Pistachio (Pistacia vera L.) is a member of family Anacardiaceae. The pistachio is deciduous and dioecious which is drought resistance and very tolerant to hot and dry weather. Area suitable for commercial olive production is generally satisfactory for pistachio. Pistachio tolerates low winter temperature but is sensitive to spring forest. April frost may fall flowers and new vegetative growth. $\backslash$ the pistachio does not tolerate high humidity in growing season (khan, 1999). In pistachio bud break, shoot elongation and leaf expansion occur during the spring, flush growth between late March and mid May (Crane and Iwakiri, 1981). the main roots grow vertically through the soil 2 to $6 \mathrm{~m}$ or more in depth which allow the plant to absorb water deep in the soil during the periods of drought (Tavallalli and Rahemi, 2007). There are about 11 specious of pistachio trees (Pistacia spp. L). P. vera is the only specious grown commercially because it produces fruit of adequate size to be marketed. Specious such as $P$. atlantica, $P$. terebinthus and $P$. integrrima are used as rootstocks for P.vera (Herra, 1997) some of these specious are used as ornamentals and some valued as fruit trees (Atli etal, 2001). Chemical composition of pistachio may vary depending upon cultivars, rootstocks and maturity and moisture content (Davarynejad etal, 2012). Magnetic water treatment remains a little known treatment in the field of plants metabolism and water relations. It is highly important is to know how magnet affects water (Khazan, 2011). The water treated by the magnetic field or pass through a magnetic device called magnetized water. It has been known that magnetic fields have effects on the normal functions of living things (Andul Qadus and Hozayn, 2010). The effects of magnetic fields on running water have been observed for years. Patents on treating water with magnets appeared as early as the 1050's in Russia, China, Poland and Bulgaria, and developed more slowly in the west (life sources, Inc.2003). Al-Kaabi (2006) recorded that the irrigation with magnetic water (500 and 1000 Gauss) significantly increased all growth parameters for mahaly orange seedlings include; plant height, stem branches, root length, root branches, dry weight of vegetative part and roots, stem diameter, total chlorophyll and total carbohydrate. Irrigation lentil plant with magnetized water significantly improvement the most studied parameters include; plant height, fresh and dry weight per plant, water content, chlorophyll, carotenoids, total pigments, total phenols and total indole for two seasons (Abdul Qados and hozayn, 2010). Hozayn etal (2010) mentioned that the magnetized water treatment exhibited an increase in the 
number of protein bands, yield and yield components, traits of monocotyledonous crops (wheat and flax) and dicotyleddonous (chick pea and lentil) compared with crop irrigated with tap water. Tayari and Jamshidi (2011) investigated that the magnetically treated water with 5000 Gauss has an enhancing effect on the photosynthetic pigments, water use efficiency and relative water content but under $30 \%$ soil water holding capacity after 6 and 8 months also the essential elements ( $\mathrm{Mg}, \mathrm{Ca}, \mathrm{P}$, and $\mathrm{K} \mathrm{ml} / \mathrm{L}$ ) were increased significantly in plants irrigated with magnetically treated water compared to their control. Sadeghipour and Aghae (2013) showed that irrigation of cowpea plants with magnetized water increased leaf, stem and root fresh and dry weights as well as total biomass, stomatal conductance water use efficiency and leaf area. Pistachio trees as most plants require 14 elements for normal growth and reproduction. These essential elements are classified as either macro nutrients $(\mathrm{N}, \mathrm{P}, \mathrm{K}, \mathrm{Ca}, \mathrm{Mg}$ and $\mathrm{S})$ or micronutrients $(\mathrm{Fe}, \mathrm{Mn}, \mathrm{Cl}, \mathrm{B}, \mathrm{Cu}, \mathrm{Zn}, \mathrm{Ni}$ and $\mathrm{Mo}$ ) based on the concentration normally present in plants, each essential for disease resistance and quality (Afrousheh etal, 2010). Boron long has been recognized as an essential element for plant growth. However, its role and mode of action are unknown. Boron has been reported to be involved in such diverse processes as nucleic acid metabolism, cell division, sugar biosynthesis and translocation and membrane functions (Patrick etal, 1995) Finally we emphasized that the technique of magnetic field in agricultural fields could be a promising technique for agricultural improvement but extensive researches is still required to emphasize in the future.

The present study is aimed to investigating the possible effects of magnetized irrigation water, NPK and Boron fertilizing on seedling growth of two Pistacia vera L. cultivars.

\section{Materials and methods}

The study was carried out at agriculture college field - Salahaddin university- Erbil, Kurdistan region of Iraq, during spring season (growth stage) of 2013. The study was done on the seedlings of two Pistacia vera L. cultivars (Kalle-Ghochi and Musilly-Mahali). The age of treated seedlings was one year cultivated in black plastic bags ( $45 \mathrm{~cm}$ length and $30 \mathrm{~cm}$ diameter) filled with sandy loem to sand clay loem soil. The seedlings were covered by white polyethylene ( $2.5 \mathrm{~m}$ height) and were fertilized with four different doses $(0,250,500$ and 1000 ppm) of NPK (20:20:20) (Demirikiranand cangiz, 2010), four different doses $(0,5,10$ and $20 \mathrm{ppm})$ of Boron as $\mathrm{H}_{3} \mathrm{BO}_{3}$ were applied. The seedlings were irrigated with four levels of magnetized water $(0,1500$ and 3000 Gauss). At the end of experimental periods the field measurements were recorded: plant height (cm), number of branches per plant, number of leaves per plant, seedling diameter $(\mathrm{mm})$, wet and dry weights of vegetative part $(\mathrm{gm})$, root length $(\mathrm{cm})$ and wet and dry weights of roots $(\mathrm{gm})$. Chlorophyll content in the leaves determined by chlorophyll meter SPAD 502 (Markwell etal, 1995; Coste etal, 2010 and Hardin etal, 2012). The evaluating of water relative content (RWC) determined by moisture meter L606 Wagner. While the leaf area was measured by digital planimeter placom KP $90 \mathrm{~N}$.

Statistical analysis of results based on factorial complete randomized design with three replications, each replication was containing two seedlings. The data were submitted to analysis of variance, the means compared by least significant differences (L.S.D.) at probability level 5\% significant using. (SAS institute, 2005)

\section{1- Effect of cultivars}

\section{Results and discussion}

Type of cultivar affected significantly on the leaf area, number of leaves and nodes, shoot diameter, shoot length, shoot wet and dry weights, root length and RWC. The higher levels of leaf area, number of leaves and nodes, shoot length and RWC $\left(18.08 \mathrm{~cm}^{2}, 24.21,41.27,44.21 \mathrm{~cm}\right.$ and $8.96 \%$ respectively) were recorded form Musilli cultivar. Best results of shoot wet and dry weights and root length $(45.37 \mathrm{gm}, 33.49 \mathrm{gm}$ and $32.95 \mathrm{~cm}$ respectively) were obtained from Kalle-Ghochi cultivar (table 1). This may indicate high genetic variability between these two cultivars.

\section{2- Effect of magnetic water}

Table (2) shows the significant effects of magnetic water in different levels on leaf area, number of nodes, shoot diameter, shoot length, root wet and dry weights and RWC. The highest value of leaf area $\left(17.35 \mathrm{~cm}^{2}\right)$ was obtained in the level of 3000 Gauss, and highest number of nodes, shoot diameter, shoot length and RWC $(43.08,6.26 \mathrm{~mm}, 39.35 \mathrm{~cm}$ and $8.93 \%$ respectivelt) were measured in the level of 1500 Gauss. However, the highest root wet and dry weights (41.29 and 33.94 gm respectively) were obtained with non-treated water.

\section{3- Effect of NPK}

NPK levels did not affect significantly on the growth parameters except leaf area and shoot wet weight when the highest leaf area $\left(25.18 \mathrm{~cm}^{2}\right)$ was measured in the control and the highest shoot weight $(41.97 \mathrm{gm})$ was recorded from 500 ppm NPK (table 3). 


\section{4- Effect of Boron}

There were no detectable differences in the growth parameters except leaf area, the highest value $(24.50$ $\mathrm{cm}^{2}$ ) was recorded from $10 \mathrm{ppm}$ Boron (table 4).

\section{5- Interaction effects of cultivars and magnetic water}

The analysis of variance showed significant differences among treatments for most traits were measured (table 5). The greater leaf area $\left(18.50 \mathrm{~cm}^{2}\right)$ was measured from the interaction of Muselli cultivar and 3000 Gauss level, and the highest number of leaves 41.20 was measured from Kalle-Ghochi cultivar and 3000 Gauss level. However, the highest values of number of nodes, shoot length and RWC $(45.06,45.08 \mathrm{~cm}$ and $9.14 \%$ respectively) were recorded from the interaction between Musilli cultivar and 1500 Gauss level. While the best results for shoot diameter, shoot wet weight and root length $(7.26 \mathrm{~mm}, 46.93 \mathrm{gm}$ and $33.76 \mathrm{~cm}$ respectively) were obtained from Kalle-Ghochi and 1500 Gauss, but shoot dry weight, root wet and dry weight $(34.61 \mathrm{gm}$, $41.60 \mathrm{gm}, 34.56 \mathrm{gm}$ respectively) recorded from the control of Kalle-Ghochi cultivar (table 5). The stimulatory effect of magnetized water on growth parameter may be attributed to the induction of cell metabolism and mitosis division (Abdol Qados and Hozayn 2010)

\section{6- Interaction effects of cultivars and NPK levels}

Table (6) shows significant differences among the average numbers of leaf area, number of leaves and nodes, shoot length, shoot weight, shoot dry weight, root wet weight and relative water content. The highest values of leaf area, number of nodes and shoot length $\left(19.06 \mathrm{~cm}^{2}, 42.57\right.$ and $44.90 \mathrm{~cm}$ respectively) were recorded from the interaction between Musilli cultivar and $500 \mathrm{ppm}$ NPK. The best results of number of leaves and shoot wet weight (43.19 and 48.08gm respectively) were obtained in the interaction of Kalle-Ghochi cultivar and $500 \mathrm{ppm}$ NPK. However, the best shoot diameter $(7.18 \mathrm{~mm})$ was recorded from the interaction of Kalle-Ghochi cultivar with 500 and $1000 \mathrm{ppm}$ NPK. While the greater values of dry weight and root length (35.58gm and $34.49 \mathrm{~cm}$ respectively) were obtained in the interaction of Kalle-Ghochi and $1000 \mathrm{ppm}$ NPK, and highest root dry weight and relative water content $(41.55 \mathrm{gm}$ and $9.11 \%$ respectively) were demonstrated from the control of Musilli cultivar, but the highest chlorophyll content in the leaves $(52.45 \%)$ was obtained from the interaction of Musilli cultivar and $1000 \mathrm{ppm}$ NPK.

\section{7- Interaction effects of cultivars and Boron}

Table (7) shows that the interaction between cultivars and Boron fertilizer increased significantly leaf area the best result $\left(20.55 \mathrm{~cm}^{2}\right)$ was observed in the Musilli and 20ppm B. While the highest number of leaves and shoot weight (40.81 and 46.23 gm respectively) were observed in control treatment of Kalle-Ghochi cultivar. Number of nodes, shoot length and RWC responses significantly, the greater averages $(42.08,44.83 \mathrm{~cm}$ and $9.04 \%$ respectively) were obtained in the Musilli cultivar when treated with $10 \mathrm{ppm}$ B. Treated seedlings of Kalle-Ghochi with 10ppm B get best average of shoot diameter $(7.19 \mathrm{~mm})$, and fertilizing the same cultivar with $5 \mathrm{ppm} \mathrm{B}$ gets the best averages of shoot dry weight, root length and root wet weight $(34.04,34.28 \mathrm{gm}$ and 40.80 gm respectively)

\section{8- Interaction effects of magnetic water and NPK}

The results in table (8) also show the promotive effects of magnetic water with NPK interaction on most of seedling growth characteristics. The best result of leaf area and RWC $\left(19.32 \mathrm{~cm}^{2}\right.$ and $9.04 \%$ respectively) were obtained in 3000 Gauss magnetic level with 500ppm NPK. While the highest number of nodes and minimum value of shoot length ( 42.19 and $36.08 \mathrm{~cm}$ respectively) were measured in the interaction between 3000 Gauss and $250 \mathrm{ppm}$ NPK, but the best average $(41.10 \mathrm{~cm}$ was obtained in the untreated seedling with NPK at the level of magnetic water about 1500 Gauss, $500 \mathrm{ppm}$ dose of NPK gave the highest value (44.87gm) of shoot wet weight without magnetic water treatment. Greatest averages of root length, root wet and dry weights $(34.00 \mathrm{~cm}$, $44.76 \mathrm{gm}$ and $36.07 \mathrm{gm}$ respectively) were obtained by untreated seedling, and the best chlorophyll content (52.49\%) was recorded from 1000ppm NPK with untreated water. Our results about root growth is reverse with the results of Sadeghipour (2013), he referred to that the stimulatory impact of magnetic water may be also ascribed to the increasing of stomatal conductance and root growth in cowpea which increase absorption and assimilation of nutrients.

\section{9- Interaction effects of magnetic water and Boron}

Regarding to the interaction effects of magnetic water levels and Boron doses on growth characteristics, data in (table 9) shows the promotive effects on some vegetative parameters but magnetic water have reverse effects of root parameters. The highest value of leaf area $\left(28.12 \mathrm{~cm}^{2}\right)$ was observed from 3000 Gauss magnetic water and 5ppm Boron, while highest number of nodes and chlorophyll content were obtained from 1500 Gauss magnetic water and 10ppm Boron. However, the other highest averages were obtained in the seedlings when 
irrigated with tap water with different doses of Boron as follows for shoot wet and dry weights $(44.39 \mathrm{gm}$ and $33.40 \mathrm{gm}$ respectively) were obtained from $20 \mathrm{ppm}$ Boron, for root length and root dry weight $(33.75 \mathrm{~cm}$ and $34.57 \mathrm{gm}$ respectively) were recorded from $5 \mathrm{ppm}$ Boron, while for root wet weight (41.18gm) was measured from 10ppm Boron. Maybe the positive effect of Boron with increasing dry weight is because of its impact on increasing activity of enzymes and supplying the products of photosynthesis, and its negative effects is because of physiological problems and protoplasm injury due to Boron toxicity (Kord etal, 2010).

\section{0- Interaction effects of NPK and Boron}

The comparison values of growth characteristics as affected by NPK and Boron interactions are present in (table 10). The best result of leaf area $\left(18.83 \mathrm{~cm}^{2}\right)$ was obtained only with $20 \mathrm{ppm}$ Boron without NPK, and the highest number of leaves (36.56) was measured by using 500ppm NPK without Boron application. However, the highest values of number of nodes and RWC (41.86 and 9.38\%) were obtained in $500 \mathrm{ppm}$ NPK and 10ppm Boron. The best shoot wet weight $(44.37 \mathrm{gm})$ was resulted untreated seedlings. While the highest root length $(31.69 \mathrm{~cm})$ was measured in $1000 \mathrm{ppm}$ NPK and $10 \mathrm{ppm}$ Boron interaction. The best root wet and dry weight $(43.63 \mathrm{gm}$ and $35.47 \mathrm{gm})$ were obtained in $500 \mathrm{ppm}$ NPK and $20 \mathrm{ppm}$ Boron, the highest chlorophyll content $(53.33 \%)$ was obtained in 1000ppm NPK and 5ppm Boron.

\section{1- Interaction effects of cultivars, magnetic water and NPK}

Results of this study show that pistachio seedling growth parameters were significantly affected with the type of cultivar, application of magnetic water and NPK interactions (table 11). The highest leaf area $\left(20.90 \mathrm{~cm}^{2}\right)$ was measured in Musilli seedlings irrigated with 3000 Gauss magnetic water at 500ppm NPK treatment, while the highest chlorophyll and RWC (54.96\% and $9.61 \%$ respectively) were obtained in the same cultivar and magnetic water application at 1000ppm NPK. The highest number of leaves and shoot wet weight (43.54 and $51.22 \mathrm{gm}$ respectively) were resulted in Kalle-Ghochi cultivar and 500ppm NPK without magnetic water application, but the greater number of nodes (46.88) was counted in the Musilli seedlings when irrigated with 1500 Gauss magnetic water and 500ppm NPK also. However, the highest value of shoot diameter $(7.55 \mathrm{~mm})$ was measured in the interaction of Kalle-Ghochi, 1500 Gauss magnetic water and 500ppm NPK. The longest seedling shoot $(48.50 \mathrm{~cm})$ was obtained in Musilli seedlings when irrigated with 1500 Gauss magnetic water treatment without NPK application. The highest dry weights of shoot and root $(37.28 \mathrm{gm}$ and $36.22 \mathrm{gm}$ respectively) the first one obtained in the Kalle-Ghochi seedlings treated with 1500 Gauss magnetic water at $1000 \mathrm{ppm}$ NPK, the second one obtained in the same cultivar and NPK dose without magnetic water irrigation, the best results of root length and root wet weight were measured in the control of both Kalle-Ghochi and Musilli cultivars respectively.

\section{2- Interaction effects of cultivars, magnetic water and Boron}

Growth characteristics response significantly to cultivar, magnetic water and Boron interactions except chlorophyll contents of leaves (table 12). The highest leaf area and shoot length were obtained in the Musilli cultivar and 10ppm Boron but in different magnetic water levels ( 0 and 1500 Gauss respectively). While the greatest leaf number $(45.00)$ and shoot diameter $(7.33 \mathrm{~mm})$ were measured for Kalle-Ghochi cultivar and 1500 Gauss magnetic water with different Boron doses (10ppm and 20ppm respectively). However, the highest number of nodes (17.21) and longest root $(35.75 \mathrm{~cm})$ were recorded in 1500 Gauss magnetic water and 20ppm Boron in different cultivars (Musilli and Kalle-Ghochi respectively). The highest shoot wet weight was obtained in Kalle-Ghochi seedlings irrigated with 1500 Gauss magnetic water without Boron application. The highest values of shoot dry weight, root wet and dry weights $(38.92 \mathrm{gm}, 45.44 \mathrm{gm}$ and $36.17 \mathrm{gm}$ respectively) resulted from the interaction between Kalle-Ghochi cultivar and 20ppm Boron irrigated with tap water. The biggest amount of RWC in the leaves $(9.28 \%$ ) was observed in Musilli seedlings when they were treated with 3000 Gauss magnetic water and 5ppm Boron.

\section{3- Interaction effects of cultivar, NPK and Boron}

Table (13) shows that the interaction of cultivars, NPK and Boron significantly affected the growth Pistacia vera $\mathrm{L}$. seedlings. The highest leaf area and root dry weight $\left(22.24 \mathrm{~cm}^{2}\right.$ and $38.46 \mathrm{gm}$ respectively) were measured in Musilli cultivar when they were treated with 500ppm NPK and 20ppm Boron. The highest number of leaves $(47.50)$, soot dry weight $(36.50 \mathrm{gm})$ and root length $(36.67 \mathrm{~cm})$ were observed in Kalle-Ghochi seedlings treated with $1000 \mathrm{ppm}$ NPK and different Boron doses $(10,20$ and $5 \mathrm{ppm}$ respectively). The best results of number of nodes and shoot length $(45.22$ and $46.33 \mathrm{~cm}$ respectively) resulted from the interaction among Musilli cultivar, 500ppm NPK and 10ppm Boron. The highest values of shoot diameter $\left(7.45 \mathrm{~cm}^{2}\right)$, shoot wet weight $(50.81 \mathrm{gm})$ and RWC $(9.67 \%)$ were resulted form Kalle-Ghochi cultivar treated with 500ppm NPK and different Boron levels (5,0 and $20 \mathrm{ppm}$ respectively). However, the highest root wet weight (46.27gm) was 
measured from untreated seedlings of Musilli cultivars, where the highest chlorophyll content (55.47\%) was resulted from the same cultivar with 1000ppm NPK and 5ppm Boron interaction treatment.

\section{4- Interaction effects of magnetic water, NPK and Boron}

Significant increasing of growth parameters were varied among the levels of magnetic water, NPK and Boron interaction treatment (table 14). The best leaf area $\left(22.07 \mathrm{~cm}^{2}\right)$ and RWC $(10.03 \%)$ resulted from the interaction between 3000 Gauss magnetic water and 500ppm NPK but in different Boron levels (5 and 10 ppm respectively). The highest number of leaves and chlorophyll content (41.75 and $57.78 \%$ respectively) were obtained in 3000 Gauss magnetic water, 1000ppm NPK and 5ppm Boron treatment. The highest number of nodes (47.50) was observed in 1500 Gauss magnetic water, 500ppm NPK and 10ppm Boron, while the highest shoot wet and dry weights $(48.89 \mathrm{gm}$ and $36.21 \mathrm{gm}$ respectively) were resulted from the interaction between $1000 \mathrm{ppm}$ NPK and 20ppm Boron without magnetic water treatment. However, the highest values of root length $(35.88 \mathrm{~cm})$ and root dry weight $(38.14 \mathrm{gm})$ were obtained from the seedling irrigated with tap water and $5 \mathrm{ppm}$ Boron in different NPK doses (500 and 1000 ppm respectively). Finally the best root wet weight was obtained in non-treating seedlings.

\section{5- Interaction effects of cultivars, magnetic water, NPK and Boron}

Results in the table (15) indicated that the interaction of both cultivars, levels of magnetic water, NPK and Boron doses have significant effects on all growth characteristics. Musilli seedlings irrigated with tap water treated with 500ppm NPK gave the highest values of leaf area and shoot length in different Boron doses (20 and $10 \mathrm{ppm}$ respectively). The highest number of leaves (51.33) was recorded from Kalle-Ghochi seedlings treated with 1500 Gauss magnetic water, 1000ppm NPK and 10ppm Boron. The thickest diameter of seedlings $(8.36 \mathrm{~mm})$ was measured from Kalle-Ghochi cultivar treating with 3000 Gauss magnetic water and 10ppm Boron. The highest shoot wet and dry weights $(59.26 \mathrm{gm}$ and $44.13 \mathrm{gm}$ respectively) were resulted from KalleGhochi seedlings irrigated with tap water treated with 1000ppm NPK and 20ppm Boron, and the longest root $(41.50 \mathrm{~cm})$ was recorded with the same above treatment but with no NPK doses. Untreated Musilli seedlings with magnetic water and NPK gave the best results of root wet and dry weights with 0 and 10ppm Boron respectively. Chlorophyll and RWC contains varied among the treatments, the best chlorophyll content $(60.85 \%)$ resulted in Kalle-Ghochi seedlings treated with 1500 Gauss magnetic water, 250ppm NPK and 10ppm Boron, while the best RWC (10.70\%) was measured from Musilli seedlings irrigated with 3000 Gauss magnetic water, 1000ppm NPK and 5ppm Boron.

In this research the observations generally indicate that the root of non treated seedlings obtained the best length and weights, because magnetic water is absorbed by the roots near the soil surface more than untreated water, so the roots are not required to grow deeply.

Table 1. Effect of (Pistacia vera L.) cultivars on growth characteristics.

\begin{tabular}{|c|c|c|c|c|c|c|c|c|c|c|c|c|}
\hline cultivars & $\begin{array}{l}\text { Leaf Area } \\
\left(\mathrm{cm}^{2}\right)\end{array}$ & No. of leaves & No. of Nods & $\begin{array}{c}\text { Shoot } \\
\text { Diameter } \\
(\mathrm{mm})\end{array}$ & $\begin{array}{c}\text { Shoot } \\
\text { Length }(\mathrm{cm})\end{array}$ & $\begin{array}{c}\text { shoot wet } \\
\text { weight }(\mathrm{gm})\end{array}$ & $\begin{array}{c}\text { shoot dry } \\
\text { weight }(\mathrm{gm})\end{array}$ & $\begin{array}{c}\text { Root Length } \\
(\mathrm{cm})\end{array}$ & $\begin{array}{c}\text { Root wet } \\
\text { weight }(\mathrm{gm})\end{array}$ & $\begin{array}{c}\text { Root dry } \\
\text { weight }(\mathrm{gm})\end{array}$ & $\begin{array}{c}\text { Chlorophyll } \\
\text { content } \%\end{array}$ & $\begin{array}{c}\text { Relative } \\
\text { water } \\
\text { content } \%\end{array}$ \\
\hline Kalle-Ghochi & 15.24 & 40.75 & 34.84 & 7.11 & 32.79 & 45.37 & 33.49 & 32.95 & 39.12 & 31.58 & 48.89 & 8.12 \\
\hline Musilli & 18.08 & 24.21 & 41.27 & 5.20 & 44.21 & 35.95 & 26.26 & 29.02 & 37.58 & 30.83 & 49.90 & 8.96 \\
\hline L.S.D $<0.05$ & 0.62 & 2.14 & 1.96 & 0.16 & 1.42 & 2.14 & 1.57 & 1.54 & N.S. & N.S. & N.S. & 0.47 \\
\hline
\end{tabular}

Table 2. Effect of magnetic water on growth characteristics of (Pistacia vera $\mathrm{L}$. .).

\begin{tabular}{|c|c|c|c|c|c|c|c|c|c|c|c|c|}
\hline $\begin{array}{c}\text { M.W. } \\
(\text { gauss })\end{array}$ & $\begin{array}{c}\text { Leaf Area } \\
\left(\mathrm{cm}^{2}\right)\end{array}$ & No. of leaves & N0. of Nods & $\begin{array}{c}\text { Shoot } \\
\text { Diameter } \\
(\mathrm{mm})\end{array}$ & $\begin{array}{c}\text { Shoot } \\
\text { Length }(\mathrm{cm})\end{array}$ & $\begin{array}{c}\text { shoot wet } \\
\text { weight }(\mathrm{gm})\end{array}$ & $\begin{array}{c}\text { shoot dry } \\
\text { weight }(\mathrm{gm})\end{array}$ & $\begin{array}{c}\text { Root Length } \\
(\mathrm{cm})\end{array}$ & $\begin{array}{c}\text { Root wet } \\
\text { weight (gm) }\end{array}$ & $\begin{array}{c}\text { Root dry } \\
\text { weight (gm) }\end{array}$ & $\begin{array}{c}\text { Chlorophyll } \\
\text { content \% }\end{array}$ & $\begin{array}{c}\text { Relative } \\
\text { water } \\
\text { content \% }\end{array}$ \\
\hline 0 & 15.62 & 31.34 & 33.91 & 6.00 & 38.92 & 41.62 & 22.75 & 31.75 & 41.29 & 33.94 & 50.721 & 7.934 \\
\hline 1500 & 17.00 & 33.81 & 43.08 & 6.26 & 39.35 & 40.77 & 29.92 & 30.81 & 36.34 & 29.08 & 36.920 & 8.925 \\
\hline 3000 & 17.35 & 32.30 & 42.18 & 6.22 & 37.24 & 39.58 & 29.00 & 30.40 & 37.43 & 30.60 & 48.757 & 8.764 \\
\hline L.S.D $<\mathbf{0 . 0 5}$ & $\mathbf{0 . 7 5}$ & N.S. & $\mathbf{2 . 4}$ & $\mathbf{0 . 2}$ & $\mathbf{1 . 7 4}$ & N.S. & N.S. & N.S. & $\mathbf{3 . 0 1}$ & $\mathbf{2 . 6 4}$ & N.S. & $\mathbf{0 . 5 8}$ \\
\hline
\end{tabular}


Table 3. Effect of NPK on growth characteristics of (Pistacia vera $\mathrm{L}_{\text {.) }}$.

\begin{tabular}{|c|c|c|c|c|c|c|c|c|c|c|c|c|}
\hline $\begin{array}{c}\text { NPK } \\
\mathrm{ppm}\end{array}$ & $\begin{array}{c}\text { Leaf Area } \\
\left(\mathrm{cm}^{2}\right)\end{array}$ & No. of leaves & N0. of Nods & $\begin{array}{c}\text { Shoot } \\
\text { Diameter } \\
(\mathrm{mm})\end{array}$ & $\begin{array}{c}\text { Shoot } \\
\text { Length }(\mathrm{cm})\end{array}$ & $\begin{array}{c}\text { shoot wet } \\
\text { weight }(\mathrm{gm})\end{array}$ & $\begin{array}{c}\text { shoot dry } \\
\text { weight }(\mathrm{gm})\end{array}$ & $\begin{array}{c}\text { Root Length } \\
(\mathrm{cm})\end{array}$ & $\begin{array}{c}\text { Root wet } \\
\text { weight }(\mathrm{gm})\end{array}$ & $\begin{array}{c}\text { Root dry } \\
\text { weight (gm) }\end{array}$ & $\begin{array}{c}\text { Chlorophyll } \\
\text { content \% }\end{array}$ & $\begin{array}{c}\text { Relative } \\
\text { water } \\
\text { content \% }\end{array}$ \\
\hline 0 & 25.18 & 32.91 & 37.30 & 6.14 & 38.71 & 40.85 & 29.90 & 31.68 & 39.64 & 31.54 & 48.42 & 8.60 \\
\hline 250 & 16.96 & 31.04 & 38.71 & 6.13 & 38.27 & 38.73 & 28.95 & 29.72 & 36.24 & 29.79 & 50.54 & 8.42 \\
\hline 500 & 17.38 & 33.72 & 38.96 & 6.13 & 38.41 & 41.97 & 29.92 & 31.03 & 39.66 & 32.26 & 47.54 & 8.68 \\
\hline 1000 & 15.51 & 32.26 & 37.26 & 6.22 & 38.08 & 41.08 & 30.72 & 31.50 & 37.88 & 31.24 & 51.10 & 8.48 \\
\hline L.S.D< $\mathbf{0 . 0 5}$ & $\mathbf{0 . 8 7}$ & N.S. & N.S. & N.S. & N.S. & $\mathbf{3 . 0 2}$ & N.S. & N.S. & N.S. & N.S. & N.S. & N.S. \\
\hline
\end{tabular}

Table 4. Effect of boron on growth characteristics of (Pistacia vera $\mathrm{L}$. .) .

\begin{tabular}{|c|c|c|c|c|c|c|c|c|c|c|c|c|}
\hline $\begin{array}{l}\text { Boron } \\
\text { ppm }\end{array}$ & $\begin{array}{l}\text { Leaf Area } \\
\left(\mathrm{cm}^{2}\right)\end{array}$ & No. of leaves & NO. of Nods & $\begin{array}{c}\text { Shoot } \\
\text { Diameter } \\
(\mathrm{mm})\end{array}$ & $\begin{array}{c}\text { Shoot } \\
\text { Length }(\mathrm{cm})\end{array}$ & $\begin{array}{c}\text { shoot wet } \\
\text { weight (gm) }\end{array}$ & $\begin{array}{c}\text { shoot dry } \\
\text { weight (gm) }\end{array}$ & $\begin{array}{l}\text { Root Length } \\
\text { (cm) }\end{array}$ & $\begin{array}{c}\text { Root wet } \\
\text { weight (gm) }\end{array}$ & $\begin{array}{c}\text { Root dry } \\
\text { weight (gm) }\end{array}$ & $\begin{array}{c}\text { Chlorophyll } \\
\text { content } \%\end{array}$ & $\begin{array}{c}\text { Relative } \\
\text { water } \\
\text { content } \%\end{array}$ \\
\hline 0 & 16.01 & 32.59 & 37.80 & 6.20 & 38.36 & 41.44 & 29.99 & 29.95 & 38.51 & 31.04 & 49.624 & 8.348 \\
\hline 5 & 16.86 & 32.19 & 38.40 & 6.13 & 36.72 & 39.51 & 29.37 & 31.27 & 37.56 & 30.78 & 49.885 & 8.371 \\
\hline 10 & 24.50 & 32.14 & 38.50 & 6.15 & 38.24 & 39.63 & 29.67 & 31.64 & 38.14 & 30.67 & 48.618 & 8.725 \\
\hline
\end{tabular}

Table 5. Interaction Effects of cultivars and magnetic water on growth characteristics of (Pistacia vera $\mathrm{L}$.).

\begin{tabular}{|c|c|c|c|c|c|c|c|c|c|c|c|c|c|}
\hline cultivars & $\begin{array}{l}\text { M.W. } \\
\text { (gauss) }\end{array}$ & $\begin{array}{l}\text { Leaf Area } \\
\qquad\left(\mathrm{cm}^{2}\right)\end{array}$ & No. of leaves & NO. of Nods & $\begin{array}{l}\text { Shoot } \\
\text { Diameter } \\
(\mathrm{mm})\end{array}$ & $\begin{array}{c}\text { Shoot } \\
\text { Length }(\mathrm{cm})\end{array}$ & $\begin{array}{c}\text { shoot wet } \\
\text { weight }(\mathrm{gm})\end{array}$ & $\begin{array}{c}\text { shoot dry } \\
\text { weight (gm) }\end{array}$ & $\begin{array}{l}\text { Root Length } \\
\text { (cm) }\end{array}$ & \begin{tabular}{|c|} 
Root wet \\
weight $(\mathrm{gm})$
\end{tabular} & $\begin{array}{c}\text { Root dry } \\
\text { weight }(\mathrm{gm})\end{array}$ & $\begin{array}{c}\text { Chlorophyll } \\
\text { content } \%\end{array}$ & $\begin{array}{c}\text { Relative } \\
\text { water } \\
\text { content } \%\end{array}$ \\
\hline \multirow{3}{*}{ Kalle-Ghochi } & 0 & 13.50 & 39.97 & 32.23 & 6.97 & 33.61 & 46.13 & 34.61 & 33.02 & 41.60 & 34.56 & 51.04 & 7.20 \\
\hline & 1500 & 16.00 & 41.09 & 35.35 & 7.26 & 33.63 & 46.93 & 34.38 & 33.76 & 38.27 & 29.83 & 48.40 & 8.71 \\
\hline & 3000 & 16.21 & 41.20 & 36.94 & 7.09 & 31.15 & 43.04 & 31.49 & 32.07 & 37.51 & 30.36 & 47.24 & 8.45 \\
\hline \multirow{3}{*}{ Musilli } & 0 & 17.73 & 22.71 & 35.59 & 5.02 & 44.22 & 37.11 & 26.82 & 30.47 & 40.98 & 33.33 & 50.40 & 8.67 \\
\hline & 1500 & 18.01 & 26.53 & 45.06 & 5.25 & 45.08 & 34.62 & 25.45 & 27.85 & 34.42 & 28.33 & 49.03 & 9.14 \\
\hline & 3000 & 18.50 & 23.40 & 43.16 & 5.34 & 43.33 & 36.12 & 26.51 & 28.72 & 37.34 & 30.84 & 50.27 & 9.08 \\
\hline \multicolumn{2}{|c|}{ L.S.D $<0.05$} & 1.45 & 3.83 & 3.33 & 0.28 & 2.43 & 3.90 & 2.65 & 2.75 & 4.04 & 3.49 & N.S. & 0.80 \\
\hline
\end{tabular}

Table 6. Interaction Effects of cultivars and NPK on growth characteristics of (Pistacia vera L.) .

\begin{tabular}{|c|c|c|c|c|c|c|c|c|c|c|c|c|c|}
\hline cultivars & $\begin{array}{l}\text { NPK } \\
\mathrm{ppm}\end{array}$ & $\begin{array}{l}\text { Leaf Area } \\
\left(\mathrm{cm}^{2}\right)\end{array}$ & No. of leaves & NO. of Nods & $\begin{array}{c}\text { Shoot } \\
\text { Diameter } \\
(\mathrm{mm})\end{array}$ & $\begin{array}{c}\text { Shoot } \\
\text { Length }(\mathrm{cm})\end{array}$ & $\begin{array}{c}\text { shoot wet } \\
\text { weight (gm) }\end{array}$ & $\begin{array}{c}\text { shoot dry } \\
\text { weight (gm) }\end{array}$ & $\begin{array}{l}\text { Root Length } \\
(\mathrm{cm})\end{array}$ & $\begin{array}{c}\text { Root wet } \\
\text { weight (gm) }\end{array}$ & $\begin{array}{c}\text { Root dry } \\
\text { weight (gm) }\end{array}$ & $\begin{array}{c}\text { Chlorophyll } \\
\text { content } \%\end{array}$ & $\begin{array}{c}\text { Relative } \\
\text { water } \\
\text { content } \%\end{array}$ \\
\hline \multirow{4}{*}{ Kalle-Ghochi } & 0 & 15.24 & 41.14 & 34.32 & 7.05 & 32.15 & 44.01 & 32.13 & 32.73 & 37.74 & 31.14 & 45.95 & 8.08 \\
\hline & 250 & 15.27 & 37.73 & 35.44 & 7.01 & 32.76 & 42.92 & 32.74 & 31.82 & 37.33 & 29.99 & 51.18 & 7.84 \\
\hline & 500 & 15.70 & 43.19 & 35.35 & 7.18 & 31.92 & 48.08 & 33.52 & 32.77 & 40.74 & 32.25 & 48.70 & 8.55 \\
\hline & 1000 & 14.73 & 40.96 & 34.25 & 7.18 & 34.34 & 46.45 & 35.58 & 34.49 & 40.69 & 32.96 & 49.75 & 8.00 \\
\hline \multirow{4}{*}{ Musilli } & 0 & 18.33 & 24.68 & 40.28 & 5.23 & 30.67 & 37.69 & 27.67 & 30.63 & 41.55 & 31.95 & 50.89 & 9.11 \\
\hline & 250 & 18.65 & 24.36 & 41.97 & 5.24 & 43.78 & 34.54 & 25.16 & 27.62 & 35.14 & 29.58 & 49.90 & 9.00 \\
\hline & 500 & 19.06 & 24.25 & 42.57 & 5.09 & 44.90 & 35.86 & 26.33 & 29.30 & 38.57 & 32.28 & 46.37 & 8.80 \\
\hline & 1000 & 16.28 & 23.56 & 40.26 & 5.26 & 42.89 & 35.72 & 25.87 & 28.51 & 35.06 & 29.53 & 52.45 & 8.95 \\
\hline \multicolumn{2}{|c|}{ L.S.D $<0.05$} & 1.69 & 4.43 & 4.11 & 0.33 & 2.85 & 4.50 & 3.1 & 3.18 & 4.70 & N.S. & 5.16 & 0.99 \\
\hline
\end{tabular}

Table 7. Interaction Effects of cultivars and boron on growth characteristics of (Pistacia vera L.).

\begin{tabular}{|c|c|c|c|c|c|c|c|c|c|c|c|c|c|}
\hline cultivars & $\begin{array}{l}\text { Boron } \\
\text { ppm }\end{array}$ & $\begin{array}{c}\text { Leaf Area } \\
\left(\mathrm{cm}^{2}\right)\end{array}$ & No. of leaves & NO. of Nods & $\begin{array}{c}\text { Shoot } \\
\text { Diameter } \\
(\mathrm{mm})\end{array}$ & $\begin{array}{c}\text { Shoot } \\
\text { Length }(\mathrm{cm})\end{array}$ & $\begin{array}{c}\text { shoot wet } \\
\text { weight (gm) }\end{array}$ & $\begin{array}{c}\text { shoot dry } \\
\text { weight (gm) }\end{array}$ & $\begin{array}{c}\text { Root Length } \\
(\mathrm{cm})\end{array}$ & $\begin{array}{c}\text { Root wet } \\
\text { weight (gm) }\end{array}$ & $\begin{array}{c}\text { Root dry } \\
\text { weight (gm) }\end{array}$ & $\begin{array}{l}\text { Chlorophyll } \\
\text { content } \%\end{array}$ & $\begin{array}{c}\text { Relative } \\
\text { water } \\
\text { content } \%\end{array}$ \\
\hline \multirow{4}{*}{ Kalle-Ghochi } & 0 & 14.44 & 40.81 & 34.75 & 7.15 & 32.15 & 46.23 & 33.01 & 30.90 & 38.33 & 31.41 & 48.15 & 7.95 \\
\hline & 5 & 15.73 & 39.92 & 34.89 & 7.07 & 33.24 & 45.61 & 34.04 & 34.28 & 40.80 & 32.61 & 48.10 & 7.76 \\
\hline & 10 & 15.19 & 42.17 & 34.92 & 7.19 & 32.18 & 45.38 & 33.46 & 33.01 & 37.94 & 30.66 & 48.16 & 8.41 \\
\hline & 20 & 15.58 & 40.13 & 34.81 & 7.02 & 34.13 & 44.24 & 33.46 & 33.62 & 39.42 & 31.65 & 51.17 & 8.37 \\
\hline \multirow{4}{*}{ Musilli } & 0 & 16.29 & 24.38 & 40.85 & 5.24 & 44.57 & 35.12 & 26.96 & 29.01 & 38.70 & 30.66 & 51.10 & 8.75 \\
\hline & 5 & 17.99 & 24.46 & 41.92 & 5.18 & 43.84 & 31.55 & 24.70 & 28.26 & 34.32 & 28.94 & 51.67 & 8.98 \\
\hline & 10 & 17.48 & 22.11 & 42.08 & 5.11 & 44.83 & 35.75 & 25.88 & 30.27 & 38.34 & 38.60 & 49.07 & 9.04 \\
\hline & 20 & 20.55 & 25.90 & 40.24 & 5.29 & 43.60 & 38.00 & 27.49 & 28.51 & 38.97 & 33.05 & 47.76 & 9.08 \\
\hline \multicolumn{2}{|c|}{ L.S.D $<0.05$} & 1.65 & 4.44 & 4.12 & 0.33 & 2.83 & 4.53 & 3.14 & 3.18 & 4.79 & N.S. & N.S. & 0.99 \\
\hline
\end{tabular}


Table 8. Interaction Effects of magnetic water and NPK on growth characteristics of (Pistacia vera $\mathrm{L}_{\text {.) }}$.

\begin{tabular}{|c|c|c|c|c|c|c|c|c|c|c|c|c|c|}
\hline $\begin{array}{l}\text { M.W. } \\
\text { (gauss) }\end{array}$ & $\begin{array}{l}\text { NPK } \\
\text { ppm }\end{array}$ & $\begin{array}{l}\text { Leaf Area } \\
\left(\mathrm{cm}^{2}\right)\end{array}$ & No. of leaves & NO. of Nods & $\begin{array}{c}\text { Shoot } \\
\text { Diameter } \\
(\mathrm{mm})\end{array}$ & $\begin{array}{c}\text { Shoot } \\
\text { Length }(\mathrm{cm})\end{array}$ & $\begin{array}{c}\text { shoot wet } \\
\text { weight }(\mathrm{gm})\end{array}$ & $\begin{array}{c}\text { shoot dry } \\
\text { weight }(\mathrm{gm})\end{array}$ & $\begin{array}{c}\text { Root Length } \\
(\mathrm{cm})\end{array}$ & $\begin{array}{c}\text { Root wet } \\
\text { weight (gm) }\end{array}$ & $\begin{array}{c}\text { Root dry } \\
\text { weight (gm) }\end{array}$ & $\begin{array}{c}\text { Chlorophyll } \\
\text { content } \%\end{array}$ & $\begin{array}{c}\text { Relative } \\
\text { water } \\
\text { content } \% \\
\end{array}$ \\
\hline \multirow{4}{*}{0} & 0 & 16.71 & 31.46 & 32.63 & 5.99 & 38.38 & 39.23 & 28.81 & 34.00 & 44.76 & 36.07 & 49.79 & 8.24 \\
\hline & 250 & 16.55 & 30.07 & 32.56 & 5.92 & 38.90 & 40.11 & 31.14 & 29.99 & 38.28 & 31.95 & 50.85 & 7.43 \\
\hline & 500 & 15.13 & 32.17 & 36.94 & 6.06 & 40.05 & 44.87 & 31.46 & 33.19 & 41.17 & 33.63 & 49.75 & 8.09 \\
\hline & 1000 & 14.07 & 31.67 & 32.42 & 6.01 & 38.34 & 42.27 & 31.44 & 29.81 & 40.96 & 34.13 & 52.49 & 7.98 \\
\hline \multirow{4}{*}{1500} & 0 & 17.41 & 34.77 & 39.90 & 6.16 & 41.10 & 31.73 & 30.66 & 30.54 & 37.56 & 30.09 & 48.83 & 8.99 \\
\hline & 250 & 16.94 & 32.81 & 41.38 & 6.20 & 38.92 & 35.85 & 28.14 & 29.04 & 34.04 & 27.82 & 50.25 & 8.99 \\
\hline & 500 & 17.68 & 35.06 & 41.48 & 6.46 & 39.10 & 42.83 & 30.85 & 31.15 & 38.79 & 30.19 & 46.68 & 8.89 \\
\hline & 1000 & 15.98 & 32.60 & 38.08 & 6.21 & 38.29 & 38.57 & 30.01 & 32.50 & 34.97 & 28.21 & 49.10 & 8.83 \\
\hline \multirow{4}{*}{3000} & 0 & 16.25 & 32.50 & 38.27 & 6.28 & 36.65 & 40.17 & 30.23 & 30.50 & 36.60 & 28.47 & 46.63 & 8.56 \\
\hline & 250 & 17.39 & 30.25 & 42.19 & 6.25 & 37.00 & 37.54 & 27.58 & 30.13 & 36.39 & 29.59 & 50.52 & 8.83 \\
\hline & 500 & 19.32 & 33.94 & 38.46 & 5.89 & 36.08 & 38.21 & 27.46 & 28.77 & 39.01 & 32.96 & 46.18 & 9.04 \\
\hline & 1000 & 16.47 & 32.50 & 41.27 & 6.44 & 39.21 & 42.41 & 30.72 & 32.19 & 37.71 & 31.38 & 51.70 & 8.63 \\
\hline \multicolumn{2}{|c|}{ L.S.D $<0.05$} & 2.17 & N.S. & 5.07 & N.S. & 4.77 & 6.42 & N.S. & 4.02 & 5.71 & 4.92 & 6.45 & 1.22 \\
\hline
\end{tabular}

Table 9. Interaction Effects of magnetic water and boron on growth characteristics of (Pistacia vera L.).

\begin{tabular}{|c|c|c|c|c|c|c|c|c|c|c|c|c|c|}
\hline $\begin{array}{l}\text { M.W. } \\
\text { (gauss) }\end{array}$ & $\begin{array}{l}\text { Boron } \\
\text { (ppm) }\end{array}$ & $\begin{array}{l}\text { Leaf Area } \\
\qquad\left(\mathrm{cm}^{2}\right)\end{array}$ & No. of leaves & NO. of Nods & $\begin{array}{c}\text { Shoot } \\
\text { Diameter } \\
(\mathrm{mm})\end{array}$ & $\begin{array}{c}\text { Shoot } \\
\text { Length }(\mathrm{cm})\end{array}$ & $\begin{array}{c}\text { shoot wet } \\
\text { weight (gm) }\end{array}$ & $\begin{array}{c}\text { shoot dry } \\
\text { weight (gm) }\end{array}$ & $\begin{array}{l}\text { Root Length } \\
(\mathrm{cm})\end{array}$ & $\begin{array}{c}\text { Root wet } \\
\text { weight }(\mathrm{gm})\end{array}$ & $\begin{array}{c}\text { Root dry } \\
\text { weight (gm) }\end{array}$ & $\begin{array}{c}\text { Chlorophyll } \\
\text { content } \%\end{array}$ & $\begin{array}{c}\text { Relative } \\
\text { water } \\
\text { content } \% \\
\end{array}$ \\
\hline \multirow{4}{*}{0} & 0 & 21.84 & 29.84 & 34.31 & 5.87 & 38.04 & 41.61 & 29.97 & 29.34 & 40.89 & 32.54 & 52.14 & 7.54 \\
\hline & 5 & 21.00 & 32.75 & 34.71 & 6.10 & 32.68 & 39.67 & 29.41 & 33.75 & 41.08 & 34.57 & 49.88 & 7.60 \\
\hline & 10 & 23.43 & 30.08 & 33.13 & 5.81 & 39.20 & 40.82 & 30.08 & 32.45 & 41.18 & 34.53 & 49.39 & 8.24 \\
\hline & 20 & 27.43 & 32.92 & 33.50 & 6.19 & 40.14 & 44.39 & 33.40 & 31.45 & 42.01 & 34.14 & 51.48 & 8.35 \\
\hline \multirow{4}{*}{1500} & 0 & 23.66 & 34.85 & 38.73 & 6.28 & 39.38 & 41.95 & 30.48 & 28.46 & 36.02 & 29.10 & 47.94 & 8.94 \\
\hline & 5 & 26.76 & 30.40 & 41.19 & 6.16 & 39.15 & 38.08 & 28.69 & 31.10 & 35.87 & 28.03 & 48.88 & 8.73 \\
\hline & 10 & 25.44 & 33.58 & 42.40 & 6.29 & 38.35 & 40.91 & 29.59 & 31.29 & 34.68 & 27.40 & 48.99 & 9.04 \\
\hline & 20 & 26.15 & 36.42 & 38.52 & 6.30 & 40.54 & 42.15 & 30.91 & 32.38 & 38.80 & 31.78 & 49.05 & 8.98 \\
\hline \multirow{4}{*}{3000} & 0 & 23.63 & 33.31 & 40.35 & 6.44 & 37.67 & 40.77 & 29.51 & 32.06 & 38.63 & 31.48 & 48.79 & 8.56 \\
\hline & 5 & 28.12 & 33.42 & 39.31 & 6.10 & 38.19 & 40.78 & 30.01 & 28.96 & 35.73 & 29.73 & 50.90 & 8.78 \\
\hline & 10 & 24.64 & 32.75 & 39.98 & 6.35 & 37.17 & 39.96 & 29.35 & 31.19 & 38.57 & 30.08 & 47.47 & 8.89 \\
\hline & 20 & 27.73 & 29.71 & 40.54 & 5.98 & 35.92 & 36.82 & 27.12 & 29.38 & 36.78 & 31.13 & 47.86 & 8.83 \\
\hline \multicolumn{2}{|c|}{ L.S.D $<0.05$} & 2.12 & N.S. & 5.12 & N.S. & N.S. & 6.49 & 4.61 & 4.02 & 5.80 & 4.96 & N.S. & 1.22 \\
\hline
\end{tabular}

Table 10. Interaction Effects of NPK and boron on growth characteristics of (Pistacia vera L.).

\begin{tabular}{|c|c|c|c|c|c|c|c|c|c|c|c|c|c|}
\hline $\begin{array}{l}\text { NPK } \\
\mathrm{ppm}\end{array}$ & $\begin{array}{l}\text { Boron } \\
\text { ppm }\end{array}$ & $\begin{array}{l}\text { Leaf Area } \\
\quad\left(\mathrm{cm}^{2}\right)\end{array}$ & No. of leaves & NO. of Nods & $\begin{array}{c}\text { Shoot } \\
\text { Diameter } \\
(\mathrm{mm})\end{array}$ & $\begin{array}{c}\text { Shoot } \\
\text { Length }(\mathrm{cm})\end{array}$ & $\begin{array}{c}\text { shoot wet } \\
\text { weight (gm) }\end{array}$ & $\begin{array}{c}\text { shoot dry } \\
\text { weight }(\mathrm{gm})\end{array}$ & $\begin{array}{l}\text { Root Length } \\
(\mathrm{cm})\end{array}$ & $\begin{array}{c}\text { Root wet } \\
\text { weight }(\mathrm{gm})\end{array}$ & $\begin{array}{c}\text { Root dry } \\
\text { weight (gm) }\end{array}$ & $\begin{array}{l}\text { Chlorophyll } \\
\text { content } \%\end{array}$ & $\begin{array}{c}\text { Relative } \\
\text { water } \\
\text { content } \%\end{array}$ \\
\hline \multirow{4}{*}{0} & 0 & 14.92 & 35.36 & 37.81 & 6.49 & 38.88 & 44.37 & 31.88 & 30.22 & 40.91 & 31.78 & 48.80 & 8.79 \\
\hline & 5 & 17.10 & 29.17 & 37.08 & 5.76 & 38.94 & 36.58 & 27.34 & 30.06 & 36.47 & 30.17 & 48.05 & 8.16 \\
\hline & 10 & 16.31 & 33.86 & 36.81 & 6.24 & 39.27 & 40.61 & 29.64 & 33.72 & 39.86 & 30.24 & 48.61 & 8.57 \\
\hline & 20 & 18.83 & 33.25 & 37.50 & 6.08 & 37.75 & 41.85 & 30.75 & 32.72 & 41.33 & 33.98 & 48.21 & 8.87 \\
\hline \multirow{4}{*}{250} & 0 & 15.26 & 32.37 & 41.22 & 6.13 & 38.97 & 39.77 & 29.23 & 29.79 & 38.95 & 31.74 & 51.66 & 8.57 \\
\hline & 5 & 17.65 & 29.94 & 38.17 & 5.76 & 37.19 & 38.76 & 28.70 & 28.49 & 35.93 & 29.41 & 51.53 & 8.66 \\
\hline & 10 & 16.97 & 28.81 & 36.92 & 6.03 & 36.17 & 39.71 & 29.57 & 28.33 & 35.28 & 28.63 & 49.22 & 8.23 \\
\hline & 20 & 17.96 & 29.67 & 36.94 & 6.16 & 39.44 & 36.69 & 28.31 & 30.40 & 34.78 & 29.37 & 49.75 & 8.22 \\
\hline \multirow{4}{*}{500} & 0 & 16.33 & 36.56 & 39.11 & 6.12 & 37.90 & 43.85 & 30.67 & 30.53 & 39.05 & 31.85 & 45.23 & 7.81 \\
\hline & 5 & 17.73 & 30.44 & 37.19 & 6.25 & 37.96 & 39.86 & 28.70 & 31.49 & 37.46 & 30.21 & 46.63 & 8.23 \\
\hline & 10 & 16.64 & 32.56 & 41.86 & 5.98 & 38.53 & 40.16 & 28.81 & 32.82 & 38.49 & 31.52 & 48.26 & 9.38 \\
\hline & 20 & 18.80 & 35.33 & 37.67 & 6.20 & 39.26 & 44.01 & 31.53 & 29.31 & 43.63 & 35.47 & 50.03 & 9.29 \\
\hline \multirow{4}{*}{1000} & 0 & 14.95 & 26.08 & 33.06 & 6.04 & 37.69 & 37.78 & 28.17 & 29.28 & 35.15 & 28.77 & 52.80 & 8.23 \\
\hline & 5 & 14.97 & 35.81 & 39.58 & 6.32 & 38.75 & 42.84 & 32.74 & 33.19 & 40.39 & 33.32 & 53.33 & 8.44 \\
\hline & 10 & 15.43 & 33.33 & 38.42 & 6.33 & 39.00 & 41.78 & 30.67 & 31.69 & 38.94 & 32.30 & 48.39 & 8.73 \\
\hline & 20 & 16.68 & 33.81 & 37.97 & 6.19 & 39.01 & 41.94 & 31.32 & 31.83 & 37.03 & 30.58 & 49.87 & 8.51 \\
\hline \multicolumn{2}{|c|}{ L.S.D $<0.05$} & 2.52 & 8.30 & 6.18 & N.S. & N.S. & 7.47 & N.S. & 4.69 & 6.77 & 5.92 & 7.48 & 1.46 \\
\hline
\end{tabular}


Table 11. Interaction Effects of cultivars, magnatic water and NPK on growth characteristics of (Pistacia vera L.).

\begin{tabular}{|c|c|c|c|c|c|c|c|c|c|c|c|c|c|c|}
\hline cultivars & $\begin{array}{l}\text { M.W. } \\
\text { (gauss) }\end{array}$ & $\begin{array}{l}\text { NPK } \\
\text { ppm }\end{array}$ & $\begin{array}{l}\text { Leaf Area } \\
\left(\mathrm{cm}^{2}\right)\end{array}$ & No. of leaves & NO. of Nods & $\begin{array}{c}\text { Shoot } \\
\text { Diameter } \\
(\mathrm{mm})\end{array}$ & $\begin{array}{c}\text { Shoot } \\
\text { Length }(\mathrm{cm})\end{array}$ & $\begin{array}{c}\text { shoot wet } \\
\text { weight }(\mathrm{gm})\end{array}$ & $\begin{array}{c}\text { shoot dry } \\
\text { weight (gm) }\end{array}$ & $\begin{array}{c}\text { Root Length } \\
(\mathrm{cm})\end{array}$ & $\begin{array}{c}\text { Root wet } \\
\text { weight (gm) }\end{array}$ & $\begin{array}{c}\text { Root dry } \\
\text { weight }(\mathrm{gm})\end{array}$ & $\begin{array}{c}\text { Chlorophyll } \\
\text { content } \%\end{array}$ & $\begin{array}{c}\text { Relative } \\
\text { water } \\
\text { content } \%\end{array}$ \\
\hline \multirow{12}{*}{ Kalle-Ghochi } & \multirow{4}{*}{0} & 0 & 15.33 & 39.38 & 33.04 & 7.06 & 32.96 & 42.63 & 31.57 & 33.69 & 41.73 & 36.06 & 49.62 & 7.24 \\
\hline & & 250 & 13.23 & 36.22 & 29.96 & 6.80 & 32.85 & 42.00 & 34.43 & 30.00 & 36.29 & 29.87 & 51.57 & 6.26 \\
\hline & & 500 & 12.03 & 43.54 & 34.96 & 7.10 & 33.56 & 51.22 & 35.65 & 35.15 & 43.70 & 36.09 & 50.80 & 7.66 \\
\hline & & 1000 & 13.43 & 40.75 & 30.96 & 6.91 & 35.06 & 48.65 & 36.78 & 33.25 & 44.68 & 36.22 & 52.16 & 7.65 \\
\hline & \multirow{4}{*}{1500} & 0 & 15.44 & 41.38 & 34.54 & 6.89 & 33.71 & 45.86 & 32.21 & 32.00 & 35.50 & 28.36 & 44.88 & 8.40 \\
\hline & & 250 & 15.62 & 36.42 & 38.33 & 7.19 & 34.58 & 44.79 & 33.22 & 35.08 & 37.97 & 30.48 & 53.74 & 8.74 \\
\hline & & 500 & 17.33 & 43.58 & 36.08 & 7.55 & 32.08 & 49.54 & 34.83 & 32.83 & 40.47 & 29.80 & 46.33 & 8.96 \\
\hline & & 1000 & 15.60 & 43.00 & 32.46 & 7.41 & 34.13 & 47.52 & 37.28 & 35.13 & 39.11 & 30.68 & 48.65 & 8.73 \\
\hline & \multirow{4}{*}{3000} & 0 & 14.96 & 42.67 & 35.38 & 7.21 & 29.79 & 43.53 & 32.63 & 32.50 & 35.98 & 28.99 & 44.88 & 8.61 \\
\hline & & 250 & 16.97 & 40.54 & 38.04 & 7.05 & 30.83 & 41.97 & 30.57 & 30.38 & 37.71 & 29.63 & 53.74 & 8.51 \\
\hline & & 500 & 17.73 & 42.46 & 35.00 & 6.88 & 30.13 & 43.48 & 30.07 & 30.33 & 38.05 & 30.85 & 46.33 & 9.04 \\
\hline & & 1000 & 15.17 & 39.13 & 39.33 & 7.23 & 33.83 & 43.19 & 32.69 & 35.08 & 38.29 & 31.98 & 48.65 & 7.64 \\
\hline \multirow{12}{*}{ Musilli } & \multirow{4}{*}{0} & 0 & 18.09 & 23.54 & 34.42 & 4.92 & 43.79 & 35.83 & 26.06 & 34.31 & 47.79 & 36.08 & 49.97 & 9.25 \\
\hline & & 250 & 19.88 & 23.92 & 35.17 & 5.05 & 44.94 & 38.22 & 27.84 & 29.98 & 40.27 & 34.03 & 50.13 & 8.60 \\
\hline & & 500 & 18.24 & 20.79 & 38.92 & 5.03 & 46.54 & 38.52 & 27.27 & 31.23 & 38.63 & 31.17 & 48.70 & 8.51 \\
\hline & & 1000 & 14.70 & 22.58 & 33.88 & 5.10 & 41.63 & 35.89 & 26.10 & 26.38 & 37.25 & 32.04 & 52.82 & 8.30 \\
\hline & \multirow{4}{*}{1500} & 0 & 19.38 & 28.17 & 45.25 & 5.42 & 48.50 & 40.44 & 29.12 & 29.08 & 39.62 & 31.81 & 52.78 & 9.58 \\
\hline & & 250 & 18.26 & 29.21 & 44.42 & 5.21 & 43.25 & 32.29 & 23.06 & 23.00 & 30.11 & 25.15 & 46.76 & 9.25 \\
\hline & & 500 & 18.03 & 26.54 & 46.88 & 5.36 & 46.13 & 36.12 & 26.88 & 29.46 & 37.12 & 30.59 & 47.03 & 8.83 \\
\hline & & 1000 & 16.37 & 22.21 & 43.71 & 5.01 & 42.46 & 29.63 & 22.74 & 29.88 & 30.82 & 25.75 & 49.56 & 8.93 \\
\hline & \multirow{4}{*}{3000} & 0 & 17.53 & 22.33 & 41.17 & 5.36 & 43.51 & 36.81 & 27.84 & 28.50 & 37.23 & 27.95 & 49.92 & 8.50 \\
\hline & & 250 & 17.81 & 19.96 & 46.33 & 5.45 & 43.17 & 33.11 & 24.59 & 29.88 & 35.06 & 29.56 & 52.82 & 9.15 \\
\hline & & 500 & 20.90 & 25.42 & 41.92 & 4.89 & 42.04 & 32.93 & 24.85 & 27.21 & 39.97 & 35.08 & 43.39 & 9.05 \\
\hline & & 1000 & 17.77 & 25.88 & 43.21 & 5.66 & 44.58 & 41.64 & 28.75 & 29.29 & 37.12 & 30.79 & 54.96 & 9.61 \\
\hline \multicolumn{3}{|c|}{ L.S.D $<0.05$} & 2.80 & 7.68 & 6.7 & 0.57 & 4.86 & 7.62 & 5.31 & 5.4 & 7.93 & 6.98 & 8.87 & 1.59 \\
\hline
\end{tabular}

Table 12. Interaction Effects of cultivars, magnatic water and boron on growth characteristics of (Pistacia vera L.).

\begin{tabular}{|c|c|c|c|c|c|c|c|c|c|c|c|c|c|c|}
\hline cultivars & $\begin{array}{l}\text { M.W. } \\
\text { (gauss) }\end{array}$ & $\begin{array}{l}\text { Boron } \\
\text { ppm }\end{array}$ & $\begin{array}{c}\text { Leaf Area } \\
\left(\mathrm{cm}^{2}\right)\end{array}$ & No. of leaves & N0. of Nods & $\begin{array}{c}\text { Shoot } \\
\text { Diameter } \\
(\mathrm{mm})\end{array}$ & $\begin{array}{c}\text { Shoot } \\
\text { Length }(\mathrm{cm})\end{array}$ & $\begin{array}{c}\text { shoot wet } \\
\text { weight (gm) }\end{array}$ & $\begin{array}{c}\text { shoot dry } \\
\text { weight (gm) }\end{array}$ & $\begin{array}{c}\text { Root Length } \\
(\mathrm{cm})\end{array}$ & $\begin{array}{c}\text { Root wet } \\
\text { weight (gm) }\end{array}$ & $\begin{array}{c}\text { Root dry } \\
\text { weight (gm) }\end{array}$ & $\begin{array}{c}\text { Chlorophyll } \\
\text { content } \%\end{array}$ & $\begin{array}{c}\text { Relative } \\
\text { water } \\
\text { content } \%\end{array}$ \\
\hline \multirow{12}{*}{ Kalle-Ghochi } & \multirow{4}{*}{0} & 0 & 14.83 & 38.05 & 33.17 & 6.90 & 32.71 & 46.72 & 33.28 & 29.98 & 40.89 & 34.75 & 52.49 & 6.70 \\
\hline & & 5 & 12.33 & 40.71 & 32.29 & 7.15 & 32.71 & 43.61 & 32.93 & 35.67 & 42.53 & 35.86 & 48.33 & 6.59 \\
\hline & & 10 & 13.58 & 38.42 & 30.08 & 6.77 & 32.96 & 44.66 & 33.31 & 32.29 & 37.55 & 31.45 & 49.31 & 7.55 \\
\hline & & 20 & 13.28 & 42.71 & 33.38 & 7.06 & 36.06 & 49.52 & 38.92 & 34.15 & 45.44 & 36.17 & 54.03 & 7.98 \\
\hline & \multirow{4}{*}{1500} & 0 & 14.15 & 40.46 & 35.25 & 7.37 & 33.29 & 48.88 & 35.04 & 29.83 & 37.84 & 30.41 & 46.30 & 8.75 \\
\hline & & 5 & 16.74 & 38.08 & 35.17 & 7.04 & 33.79 & 45.72 & 34.55 & 35.75 & 39.68 & 29.72 & 48.12 & 8.40 \\
\hline & & 10 & 16.84 & 45.00 & 37.88 & 7.30 & 31.83 & 48.40 & 34.94 & 33.88 & 37.14 & 28.70 & 47.94 & 8.95 \\
\hline & & 20 & 16.26 & 40.83 & 33.13 & 7.33 & 35.58 & 44.70 & 33.01 & 35.58 & 38.41 & 30.49 & 51.22 & 8.73 \\
\hline & \multirow{4}{*}{3000} & 0 & 14.33 & 43.92 & 35.83 & 7.18 & 30.46 & 43.08 & 30.70 & 32.88 & 36.27 & 29.08 & 45.65 & 8.39 \\
\hline & & 5 & 18.13 & 40.96 & 37.21 & 7.03 & 33.21 & 47.51 & 34.65 & 31.42 & 40.20 & 32.25 & 47.84 & 8.29 \\
\hline & & 10 & 15.15 & 43.08 & 36.79 & 7.50 & 30.17 & 43.08 & 32.14 & 32.88 & 39.14 & 31.84 & 47.23 & 8.73 \\
\hline & & 20 & 17.21 & 36.83 & 37.92 & 6.67 & 30.75 & 38.51 & 28.45 & 31.13 & 34.42 & 28.29 & 48.25 & 8.40 \\
\hline
\end{tabular}


Influence of magnetized water and some fertilizers on the growth of two cultivars of (Pistacia vera L.)

\begin{tabular}{|c|c|c|c|c|c|c|c|c|c|c|c|c|c|c|}
\hline \multirow{12}{*}{ Musilli } & \multirow{4}{*}{0} & 0 & 14.29 & 21.17 & 35.46 & 4.83 & 43.38 & 36.50 & 26.66 & 28.71 & 40.90 & 30.33 & 51.78 & 8.39 \\
\hline & & 5 & 15.68 & 24.79 & 37.13 & 5.09 & 43.85 & 35.72 & 25.89 & 31.83 & 39.64 & 33.28 & 51.44 & 8.61 \\
\hline & & 10 & 17.66 & 21.75 & 36.17 & 4.85 & 45.44 & 36.98 & 26.85 & 32.60 & 44.82 & 37.61 & 49.47 & 8.94 \\
\hline & & 20 & 23.28 & 23.13 & 33.63 & 5.32 & 44.23 & 39.26 & 27.87 & 28.75 & 38.58 & 32.12 & 48.93 & 8.73 \\
\hline & \multirow{4}{*}{1500} & 0 & 17.40 & 29.25 & 42.21 & 5.19 & 45.46 & 35.02 & 25.91 & 27.08 & 34.21 & 27.78 & 49.58 & 9.14 \\
\hline & & 5 & 18.93 & 22.71 & 47.21 & 5.27 & 44.50 & 30.44 & 22.84 & 26.46 & 32.05 & 26.35 & 49.63 & 9.06 \\
\hline & & 10 & 17.08 & 22.17 & 46.92 & 5.27 & 44.88 & 33.42 & 24.23 & 28.71 & 32.22 & 26.11 & 50.04 & 9.14 \\
\hline & & 20 & 18.61 & 32.00 & 43.92 & 5.28 & 45.50 & 39.60 & 28.81 & 29.17 & 39.18 & 33.07 & 46.88 & 9.24 \\
\hline & \multirow{4}{*}{3000} & 0 & 17.18 & 22.71 & 44.88 & 5.70 & 44.88 & 38.45 & 28.32 & 31.25 & 40.99 & 33.87 & 51.94 & 8.73 \\
\hline & & 5 & 19.36 & 25.88 & 41.42 & 5.16 & 43.17 & 34.06 & 25.37 & 26.50 & 31.27 & 27.20 & 53.95 & 9.28 \\
\hline & & 10 & 17.71 & 22.42 & 43.17 & 5.20 & 44.18 & 36.84 & 26.55 & 29.50 & 37.99 & 28.33 & 47.71 & 9.05 \\
\hline & & 20 & 19.77 & 22.58 & 43.17 & 5.29 & 41.08 & 35.14 & 25.79 & 27.63 & 39.13 & 33.98 & 47.48 & 9.26 \\
\hline \multicolumn{3}{|c|}{ L.S.D $<0.05$} & 2.63 & 7.62 & 6.77 & 0.57 & 4.93 & 7.81 & 5.33 & 5.49 & 8.04 & 6.93 & N.S. & 1.64 \\
\hline cultivars & $\begin{array}{l}\text { NPK } \\
\text { ppm }\end{array}$ & $\begin{array}{l}\text { Boron } \\
\text { ppm }\end{array}$ & $\begin{array}{c}\text { Leaf Area } \\
\left(\mathrm{cm}^{2}\right)\end{array}$ & No. of leaves & NO. of Nods & $\begin{array}{c}\text { Shoot } \\
\text { Diameter } \\
(\mathrm{mm}) \\
\end{array}$ & $\begin{array}{c}\text { Shoot } \\
\text { Length }(\mathrm{cm})\end{array}$ & $\begin{array}{c}\text { shoot wet } \\
\text { weight }(\mathrm{gm})\end{array}$ & $\begin{array}{c}\text { shoot dry } \\
\text { weight (gm) }\end{array}$ & $\begin{array}{c}\text { Root Length } \\
(\mathrm{cm})\end{array}$ & $\begin{array}{c}\text { Root wet } \\
\text { weight }(\mathrm{gm})\end{array}$ & $\begin{array}{c}\text { Root dry } \\
\text { weight (gm) }\end{array}$ & $\begin{array}{c}\text { Chlorophyll } \\
\text { content \% }\end{array}$ & 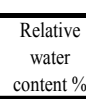 \\
\hline \multirow{16}{*}{ Kalle-Ghochi } & \multirow{4}{*}{0} & 0 & 12.87 & 43.11 & 34.61 & 7.28 & 31.53 & 46.40 & 32.11 & 29.64 & 35.54 & 30.92 & 42.71 & 8.67 \\
\hline & & 5 & 16.87 & 38.94 & 32.39 & 6.59 & 33.78 & 40.74 & 30.27 & 31.44 & 36.80 & 30.16 & 44.08 & 7.37 \\
\hline & & 10 & 14.78 & 44.67 & 34.56 & 7.36 & 32.53 & 47.40 & 34.37 & 34.39 & 38.63 & 31.04 & 48.56 & 8.22 \\
\hline & & 20 & 16.47 & 37.83 & 35.72 & 7.00 & 30.78 & 41.50 & 31.79 & 35.44 & 39.97 & 32.43 & 48.44 & 8.08 \\
\hline & \multirow{4}{*}{250} & 0 & 14.17 & 39.40 & 37.83 & 6.96 & 31.97 & 43.69 & 32.44 & 30.75 & 38.55 & 30.36 & 53.35 & 7.93 \\
\hline & & 5 & 15.12 & 41.83 & 36.61 & 7.20 & 32.72 & 46.44 & 34.29 & 34.28 & 40.34 & 32.58 & 48.58 & 7.93 \\
\hline & & 10 & 15.58 & 40.22 & 37.17 & 7.02 & 28.17 & 42.91 & 32.00 & 27.22 & 34.55 & 27.87 & 47.93 & 7.83 \\
\hline & & 20 & 16.21 & 34.72 & 34.33 & 6.94 & 35.33 & 38.66 & 32.22 & 31.97 & 35.87 & 29.15 & 54.84 & 7.65 \\
\hline & \multirow{4}{*}{500} & 0 & 15.01 & 46.28 & 37.00 & 7.20 & 32.08 & 50.81 & 34.78 & 32.17 & 40.16 & 32.55 & 44.80 & 7.10 \\
\hline & & 5 & 16.44 & 39.83 & 33.56 & 7.45 & 31.56 & 49.91 & 34.68 & 34.72 & 41.40 & 31.53 & 48.52 & 8.23 \\
\hline & & 10 & 15.98 & 41.56 & 38.50 & 7.07 & 30.72 & 43.16 & 31.28 & 33.22 & 40.05 & 32.41 & 48.24 & 9.22 \\
\hline & & 20 & 15.36 & 45.11 & 32.33 & 6.97 & 33.33 & 48.44 & 33.34 & 30.97 & 41.36 & 32.49 & 53.23 & 9.67 \\
\hline & \multirow{4}{*}{1000} & 0 & 15.71 & 34.44 & 29.56 & 7.17 & 33.03 & 44.02 & 32.72 & 31.03 & 39.08 & 31.82 & 51.73 & 8.08 \\
\hline & & 5 & 14.50 & 39.06 & 37.00 & 7.05 & 34.89 & 45.36 & 36.92 & 36.67 & 44.68 & 36.16 & 51.19 & 7.50 \\
\hline & & 10 & 14.42 & 47.50 & 33.61 & 7.36 & 32.36 & 48.05 & 36.20 & 34.17 & 38.53 & 31.33 & 47.92 & 8.37 \\
\hline & & 20 & 14.30 & 42.83 & 36.83 & 7.15 & 37.08 & 48.37 & 36.50 & 36.08 & 40.49 & 32.53 & 48.15 & 8.07 \\
\hline \multirow{16}{*}{ Musilli } & \multirow{4}{*}{0} & 0 & 16.97 & 27.61 & 41.00 & 5.70 & 46.22 & 42.35 & 31.65 & 30.81 & 46.27 & 32.64 & 54.89 & 8.92 \\
\hline & & 5 & 17.33 & 20.11 & 42.22 & 4.99 & 44.73 & 32.41 & 24.42 & 30.39 & 36.13 & 30.17 & 52.02 & 8.95 \\
\hline & & 10 & 17.83 & 23.06 & 39.06 & 5.13 & 46.01 & 33.81 & 24.91 & 33.06 & 41.09 & 29.45 & 48.66 & 8.92 \\
\hline & & 20 & 21.20 & 28.67 & 39.28 & 5.16 & 44.72 & 42.21 & 29.71 & 30.00 & 42.69 & 35.54 & 47.98 & 9.65 \\
\hline & \multirow{4}{*}{250} & 0 & 16.34 & 25.33 & 44.61 & 5.31 & 45.97 & 35.85 & 26.01 & 28.83 & 39.35 & 33.12 & 49.98 & 9.20 \\
\hline & & 5 & 20.18 & 24.83 & 42.89 & 5.15 & 44.28 & 31.08 & 23.11 & 26.42 & 31.52 & 26.24 & 54.47 & 9.38 \\
\hline & & 10 & 18.36 & 22.67 & 40.83 & 5.10 & 41.33 & 36.51 & 27.13 & 26.39 & 36.01 & 29.38 & 50.50 & 8.63 \\
\hline & & 20 & 19.71 & 24.61 & 39.56 & 5.38 & 43.56 & 34.72 & 24.40 & 28.83 & 33.70 & 29.59 & 44.66 & 8.78 \\
\hline & \multirow{4}{*}{500} & 0 & 17.66 & 26.83 & 41.22 & 5.03 & 43.72 & 36.88 & 26.57 & 28.89 & 37.95 & 31.15 & 45.67 & 8.52 \\
\hline & & 5 & 19.01 & 21.06 & 40.83 & 5.04 & 44.36 & 29.82 & 22.71 & 28.25 & 33.52 & 28.88 & 44.74 & 8.22 \\
\hline & & 10 & 17.31 & 23.56 & 45.22 & 4.89 & 46.33 & 37.15 & 26.34 & 32.42 & 36.93 & 30.62 & 48.27 & 9.53 \\
\hline & & 20 & 22.24 & 25.56 & 43.00 & 5.42 & 45.19 & 39.58 & 29.71 & 27.64 & 45.89 & 38.46 & 46.82 & 8.92 \\
\hline & \multirow{4}{*}{1000} & 0 & 14.19 & 17.72 & 36.56 & 4.92 & 42.36 & 31.54 & 23.63 & 27.53 & 31.22 & 25.73 & 53.87 & 8.37 \\
\hline & & 5 & 15.43 & 32.56 & 42.17 & 5.58 & 42.61 & 40.31 & 28.55 & 29.72 & 36.10 & 30.48 & 55.47 & 9.38 \\
\hline & & 10 & 16.43 & 19.17 & 43.22 & 5.30 & 45.64 & 35.51 & 25.14 & 29.22 & 39.35 & 33.28 & 48.87 & 9.08 \\
\hline & & 20 & 19.06 & 24.78 & 39.11 & 5.23 & 40.94 & 35.51 & 26.15 & 27.58 & 33.58 & 28.63 & 51.58 & 8.95 \\
\hline \multicolumn{3}{|c|}{ L.S.D $<0.05$} & 3.26 & 8.57 & 8.37 & 0.67 & 5.73 & 8.84 & 6.23 & 6.43 & 9.43 & 8.50 & 10.20 & 1.97 \\
\hline
\end{tabular}


Table 14. Interaction Effects of magnetic water, NPK and boron on growth characteristics of (Pistacia vera L.) .

\begin{tabular}{|c|c|c|c|c|c|c|c|c|c|c|c|c|c|}
\hline $\begin{array}{l}\text { M.W. } \\
\text { (gauss) }\end{array}$ & $\begin{array}{l}\text { NPK } \\
\text { ppm }\end{array}$ & $\begin{array}{l}\text { Boron } \\
\text { ppm }\end{array}$ & $\begin{array}{l}\text { Leaf Area } \\
\left(\mathrm{cm}^{2}\right)\end{array}$ & No. of leaves & NO. of Nods & $\begin{array}{c}\text { Shoot } \\
\text { Diameter } \\
(\mathrm{mm}) \\
\end{array}$ & $\begin{array}{c}\text { Shoot } \\
\text { Length }(\mathrm{cm})\end{array}$ & $\begin{array}{c}\text { shoot wet } \\
\text { weight }(\mathrm{gm})\end{array}$ & $\begin{array}{c}\text { shoot dry } \\
\text { weight (gm) }\end{array}$ & $\left|\begin{array}{c}\text { Root Length } \\
(\mathrm{cm})\end{array}\right|$ & $\begin{array}{c}\text { Root wet } \\
\text { weight }(\mathrm{gm})\end{array}$ & $\begin{array}{c}\text { Root dry } \\
\text { weight (gm) }\end{array}$ & $\begin{array}{c}\text { Chlorophyll } \\
\text { content \% }\end{array}$ \\
\hline \multirow{16}{*}{0} & \multirow{4}{*}{0} & 0 & 15.90 & 30.42 & 30.67 & 6.43 & 39.79 & 47.04 & 33.32 & 33.33 & 50.15 & 36.90 & 49.79 \\
\hline & & 5 & 15.92 & 31.83 & 37.75 & 5.69 & 39.33 & 31.55 & 23.68 & 32.67 & 39.75 & 32.94 & 49.30 \\
\hline & & 10 & 17.50 & 33.58 & 33.75 & 5.87 & 36.54 & 37.41 & 26.67 & 33.25 & 44.18 & 37.24 & 46.98 \\
\hline & & 20 & 17.53 & 30.00 & 32.75 & 5.98 & 37.83 & 40.93 & 31.57 & 36.75 & 44.96 & 37.20 & 53.11 \\
\hline & \multirow{4}{*}{250} & 0 & 12.07 & 32.27 & 37.00 & 5.79 & 39.42 & 41.17 & 30.63 & 27.38 & 39.62 & 32.40 & 54.55 \\
\hline & & 5 & 17.08 & 34.75 & 36.92 & 6.04 & 38.50 & 40.55 & 31.01 & 31.96 & 38.73 & 32.71 & 45.18 \\
\hline & & 10 & 17.13 & 22.83 & 27.08 & 5.50 & 37.83 & 39.78 & 30.48 & 33.67 & 35.83 & 29.66 & 52.13 \\
\hline & & 20 & 19.92 & 30.42 & 29.25 & 6.36 & 39.83 & 38.96 & 32.43 & 26.96 & 38.94 & 33.04 & 51.53 \\
\hline & \multirow{4}{*}{500} & 0 & 16.33 & 32.42 & 36.92 & 5.87 & 36.71 & 43.12 & 29.29 & 29.08 & 38.17 & 31.56 & 46.65 \\
\hline & & 5 & 11.45 & 31.25 & 33.33 & 6.38 & 39.04 & 43.98 & 31.88 & 35.88 & 40.41 & 34.49 & 51.65 \\
\hline & & 10 & 13.55 & 31.25 & 39.58 & 5.81 & 43.42 & 43.60 & 31.30 & 35.96 & 42.76 & 34.93 & 49.49 \\
\hline & & 20 & 19.20 & 33.75 & 37.92 & 6.19 & 41.03 & 48.79 & 33.38 & 31.83 & 43.32 & 33.53 & 51.22 \\
\hline & \multirow{4}{*}{1000} & 0 & 13.95 & 23.33 & 32.67 & 5.37 & 36.25 & 35.11 & 26.64 & 27.58 & 35.63 & 29.30 & 57.55 \\
\hline & & 5 & 11.55 & 33.17 & 30.83 & 6.37 & 36.25 & 42.59 & 31.06 & 34.50 & 45.45 & 38.14 & 53.39 \\
\hline & & 10 & 14.28 & 32.67 & 32.08 & 6.05 & 39.00 & 42.48 & 31.86 & 26.92 & 41.97 & 36.29 & 48.98 \\
\hline & & 20 & 16.48 & 37.50 & 34.08 & 6.22 & 41.88 & 48.89 & 36.21 & 30.25 & 40.81 & 32.80 & 50.04 \\
\hline \multirow{16}{*}{1500} & \multirow{4}{*}{0} & 0 & 14.92 & 39.17 & 42.25 & 6.28 & 39.83 & 44.92 & 32.31 & 23.83 & 35.20 & 28.45 & 48.56 \\
\hline & & 5 & 18.40 & 27.92 & 40.33 & 5.96 & 42.33 & 39.69 & 28.39 & 28.08 & 34.70 & 28.39 & 50.23 \\
\hline & & 10 & 16.15 & 34.00 & 40.92 & 6.19 & 43.00 & 42.95 & 31.14 & 36.50 & 36.66 & 28.29 & 49.72 \\
\hline & & 20 & 20.17 & 38.00 & 36.08 & 6.19 & 39.25 & 45.04 & 30.82 & 33.75 & 43.67 & 35.24 & 46.80 \\
\hline & \multirow{4}{*}{250} & 0 & 16.63 & 34.92 & 40.33 & 6.01 & 36.92 & 36.65 & 26.11 & 27.58 & 34.03 & 28.55 & 48.11 \\
\hline & & 5 & 17.80 & 33.50 & 43.25 & 6.35 & 41.58 & 39.87 & 28.20 & 30.42 & 35.76 & 28.56 & 53.86 \\
\hline & & 10 & 18.33 & 31.00 & 42.33 & 6.39 & 36.42 & 41.42 & 30.55 & 25.75 & 33.77 & 26.44 & 48.02 \\
\hline & & 20 & 14.98 & 31.83 & 39.58 & 6.05 & 40.75 & 36.22 & 27.70 & 32.42 & 32.62 & 27.72 & 51.02 \\
\hline & \multirow{4}{*}{500} & 0 & 15.73 & 40.25 & 41.42 & 6.47 & 40.92 & 46.95 & 33.56 & 32.83 & 42.08 & 33.61 & 47.02 \\
\hline & & 5 & 19.67 & 13.83 & 20.33 & 3.18 & 18.33 & 37.36 & 28.00 & 15.46 & 35.82 & 25.95 & 42.58 \\
\hline & & 10 & 17.82 & 34.25 & 47.50 & 6.27 & 37.50 & 40.69 & 27.85 & 30.33 & 35.19 & 28.09 & 46.81 \\
\hline & & 20 & 17.50 & 38.08 & 36.33 & 6.73 & 41.33 & 46.32 & 34.01 & 30.50 & 42.09 & 33.13 & 50.31 \\
\hline & \multirow{4}{*}{1000} & 0 & 15.82 & 25.08 & 30.92 & 6.35 & 39.83 & 39.29 & 29.93 & 29.58 & 32.78 & 25.78 & 48.09 \\
\hline & & 5 & 15.48 & 32.50 & 40.50 & 5.96 & 36.00 & 35.39 & 30.18 & 35.00 & 37.18 & 29.25 & 48.83 \\
\hline & & 10 & 15.55 & 35.08 & 38.83 & 6.30 & 36.50 & 38.60 & 28.82 & 32.58 & 33.10 & 26.79 & 51.42 \\
\hline & & 20 & 17.08 & 37.75 & 42.08 & 6.23 & 40.83 & 41.03 & 31.12 & 32.83 & 36.80 & 31.04 & 48.08 \\
\hline \multirow{16}{*}{3000} & \multirow{4}{*}{0} & 0 & 13.93 & 36.50 & 40.50 & 6.76 & 37.00 & 41.15 & 30.01 & 33.50 & 37.37 & 30.00 & 48.05 \\
\hline & & 5 & 16.98 & 31.75 & 39.08 & 6.14 & 35.42 & 38.49 & 29.95 & 28.67 & 34.95 & 29.17 & 44.63 \\
\hline & & 10 & 15.27 & 34.00 & 35.75 & 6.68 & 38.27 & 41.46 & 31.11 & 31.42 & 38.75 & 25.21 & 49.13 \\
\hline & & 20 & 18.80 & 31.75 & 43.67 & 6.07 & 36.17 & 39.59 & 29.86 & 27.67 & 35.36 & 29.52 & 44.73 \\
\hline & \multirow{4}{*}{250} & 0 & 17.07 & 29.92 & 46.33 & 6.60 & 40.58 & 41.50 & 30.95 & 34.42 & 43.20 & 34.28 & 52.33 \\
\hline & & 5 & 18.07 & 31.75 & 39.08 & 6.14 & 35.42 & 35.86 & 26.90 & 28.67 & 33.30 & 26.97 & 55.53 \\
\hline & & 10 & 15.43 & 32.58 & 41.33 & 6.20 & 34.25 & 37.94 & 27.68 & 25.58 & 36.25 & 29.78 & 47.51 \\
\hline & & 20 & 18.98 & 26.75 & 42.00 & 6.07 & 37.75 & 34.88 & 24.80 & 31.83 & 32.79 & 27.36 & 46.70 \\
\hline & \multirow{4}{*}{500} & 0 & 16.93 & 37.00 & 39.00 & 6.01 & 36.08 & 41.47 & 29.16 & 29.67 & 36.90 & 30.39 & 42.03 \\
\hline & & 5 & 22.07 & 32.42 & 37.58 & 5.99 & 38.17 & 38.26 & 26.21 & 27.67 & 36.15 & 30.19 & 45.66 \\
\hline & & 10 & 18.57 & 32.17 & 38.50 & 5.87 & 34.67 & 36.18 & 27.28 & 32.17 & 37.52 & 31.53 & 48.47 \\
\hline & & 20 & 19.70 & 34.17 & 38.75 & 5.67 & 35.42 & 36.92 & 27.19 & 25.58 & 45.47 & 39.76 & 48.55 \\
\hline & \multirow{4}{*}{1000} & 0 & 15.08 & 29.83 & 35.58 & 6.41 & 37.00 & 38.94 & 27.94 & 30.67 & 37.05 & 31.24 & 52.76 \\
\hline & & 5 & 17.87 & 41.75 & 47.42 & 6.62 & 44.00 & 50.53 & 36.97 & 30.08 & 38.53 & 32.57 & 57.78 \\
\hline & & 10 & 16.45 & 32.25 & 44.33 & 6.65 & 41.50 & 44.26 & 31.33 & 35.58 & 41.75 & 33.83 & 44.78 \\
\hline & & 20 & 16.47 & 26.17 & 37.75 & 6.10 & 34.33 & 35.91 & 26.64 & 32.42 & 33.50 & 27.90 & 51.48 \\
\hline \multicolumn{3}{|c|}{ L.S.D $<0.05$} & 4.10 & 14.79 & 10.16 & N.S. & N.S. & 13.33 & 9.61 & 7.93 & 11.88 & 10.26 & 13.16 \\
\hline
\end{tabular}


Table 15. Interaction Effects of cultivars, magnetic water, NPK and boron on growth characteristics of (Pistacia vera L.).

\begin{tabular}{|c|c|c|c|c|c|c|c|c|c|c|c|c|c|c|c|}
\hline cultivars & $\begin{array}{l}\text { M.W. } \\
\text { (gauss) }\end{array}$ & $\begin{array}{c}\text { NPK } \\
\text { ppm }\end{array}$ & $\begin{array}{l}\text { Boron } \\
\text { ppm }\end{array}$ & $\begin{array}{l}\text { Leaf Area } \\
\left(\mathrm{cm}^{2}\right)\end{array}$ & No. of leaves & N0. of Nods & $\begin{array}{c}\text { Shoot } \\
\text { Diameter } \\
(\mathrm{mm})\end{array}$ & $\begin{array}{c}\text { Shoot } \\
\text { Length }(\mathrm{cm})\end{array}$ & $\begin{array}{l}\text { shoot wet } \\
\text { weight (gm) }\end{array}$ & $\begin{array}{c}\text { shoot dry } \\
\text { weight (gm) }\end{array}$ & $\begin{array}{c}\text { Root Length } \\
(\mathrm{cm})\end{array}$ & $\begin{array}{c}\text { Root wet } \\
\text { weight (gm) }\end{array}$ & $\begin{array}{c}\text { Root dry } \\
\text { weight (gm) }\end{array}$ & $\begin{array}{c}\text { Chlorophyll } \\
\text { content } \%\end{array}$ & $\begin{array}{c}\text { Relative } \\
\text { water } \\
\text { content } \%\end{array}$ \\
\hline \multirow{48}{*}{ Kalle-Ghochi } & \multirow{16}{*}{0} & \multirow{4}{*}{0} & 0 & 17.13 & 33.17 & 28.67 & 7.57 & 33.75 & 47.36 & 32.52 & 30.25 & 42.67 & 39.86 & 50.10 & 7.25 \\
\hline & & & 5 & 15.10 & 45.00 & 37.33 & 6.71 & 35.17 & 32.90 & 24.89 & 32.33 & 37.52 & 31.78 & 46.33 & 6.40 \\
\hline & & & 10 & 15.97 & 42.33 & 31.83 & 6.77 & 29.92 & 43.84 & 31.96 & 30.67 & 36.52 & 31.05 & 51.27 & 7.65 \\
\hline & & & 20 & 13.13 & 37.00 & 34.33 & 7.21 & 33.00 & 46.44 & 36.93 & 41.50 & 50.21 & 41.55 & 50.77 & 7.65 \\
\hline & & \multirow{4}{*}{250} & 0 & 9.67 & 44.70 & 37.83 & 6.70 & 32.42 & 46.03 & 34.59 & 23.92 & 36.11 & 28.72 & 59.13 & 6.35 \\
\hline & & & 5 & 13.03 & 39.00 & 33.17 & 7.01 & 32.17 & 39.97 & 32.74 & 33.67 & 39.60 & 34.00 & 41.98 & 6.35 \\
\hline & & & 10 & 15.67 & 25.67 & 23.00 & 6.66 & 35.83 & 45.51 & 33.22 & 34.17 & 31.64 & 26.16 & 48.42 & 5.55 \\
\hline & & & 20 & 14.53 & 35.50 & 25.83 & 6.84 & 31.00 & 36.52 & 37.19 & 28.25 & 37.82 & 30.61 & 56.75 & 6.80 \\
\hline & & \multirow{4}{*}{500} & 0 & 16.67 & 44.83 & 34.83 & 6.99 & 31.25 & 50.64 & 33.78 & 35.50 & 41.47 & 35.24 & 44.47 & 5.55 \\
\hline & & & 5 & 10.20 & 41.17 & 32.00 & 7.70 & 31.50 & 56.03 & 39.46 & 37.17 & 43.92 & 37.47 & 55.92 & 7.65 \\
\hline & & & 10 & 10.37 & 39.83 & 36.33 & 6.67 & 33.00 & 42.37 & 31.92 & 37.83 & 41.98 & 35.28 & 48.78 & 8.50 \\
\hline & & & 20 & 10.87 & 48.33 & 36.67 & 7.03 & 38.50 & 55.86 & 37.46 & 30.08 & 47.44 & 36.36 & 54.05 & 8.95 \\
\hline & & \multirow{4}{*}{1000} & 0 & 15.87 & 29.50 & 31.33 & 6.33 & 33.42 & 42.87 & 32.26 & 30.25 & 43.31 & 35.19 & 56.25 & 7.65 \\
\hline & & & 5 & 10.97 & 37.67 & 26.67 & 7.19 & 32.00 & 45.55 & 34.63 & 39.50 & 49.08 & 40.20 & 49.07 & 5.95 \\
\hline & & & 10 & 12.30 & 45.83 & 29.17 & 6.98 & 33.08 & 46.92 & 36.13 & 26.50 & 40.07 & 33.33 & 48.78 & 8.50 \\
\hline & & & 20 & 14.60 & 50.00 & 36.67 & 7.15 & 41.75 & 59.26 & 44.13 & 36.75 & 46.28 & 36.17 & 54.53 & 8.50 \\
\hline & \multirow{16}{*}{1500} & \multirow{4}{*}{0} & 0 & 9.40 & 45.33 & 36.83 & 6.97 & 30.67 & 49.05 & 33.59 & 25.67 & 30.76 & 25.12 & 38.18 & 8.95 \\
\hline & & & 5 & 19.03 & 35.00 & 29.33 & 6.62 & 37.50 & 45.15 & 31.06 & 27.67 & 36.01 & 28.92 & 46.32 & 8.50 \\
\hline & & & 10 & 16.53 & 44.50 & 37.50 & 6.93 & 34.67 & 47.66 & 33.83 & 39.33 & 37.53 & 28.86 & 47.12 & 8.50 \\
\hline & & & 20 & 16.80 & 40.67 & 34.50 & 7.04 & 32.00 & 41.59 & 30.35 & 35.33 & 37.72 & 30.56 & 47.88 & 7.65 \\
\hline & & \multirow{4}{*}{250} & 0 & 15.07 & 33.17 & 36.83 & 6.84 & 33.33 & 40.78 & 29.86 & 32.33 & 39.40 & 32.42 & 52.67 & 9.40 \\
\hline & & & 5 & 15.43 & 41.50 & 41.67 & 7.47 & 35.17 & 50.85 & 35.46 & 40.17 & 40.89 & 32.25 & 53.77 & 7.65 \\
\hline & & & 10 & 17.03 & 37.67 & 40.50 & 7.38 & 29.83 & 46.37 & 35.33 & 31.67 & 36.93 & 28.77 & 47.68 & 9.85 \\
\hline & & & 20 & 14.93 & 33.33 & 34.33 & 7.07 & 40.00 & 41.17 & 32.23 & 36.17 & 34.68 & 28.48 & 60.85 & 8.05 \\
\hline & & \multirow{4}{*}{500} & 0 & 15.63 & 48.67 & 42.50 & 8.02 & 34.33 & 57.60 & 40.43 & 32.33 & 45.22 & 36.15 & 44.95 & 7.25 \\
\hline & & & 5 & 18.87 & 33.83 & 30.67 & 7.09 & 30.50 & 42.80 & 32.33 & 36.50 & 37.81 & 23.52 & 45.83 & 8.10 \\
\hline & & & 10 & 17.87 & 46.50 & 44.17 & 7.34 & 32.33 & 49.42 & 33.32 & 28.33 & 37.79 & 28.26 & 43.68 & 9.35 \\
\hline & & & 20 & 16.97 & 45.33 & 27.00 & 7.75 & 31.17 & 48.33 & 33.25 & 34.17 & 41.08 & 31.27 & 50.83 & 11.15 \\
\hline & & \multirow{4}{*}{1000} & 0 & 16.50 & 34.67 & 24.83 & 7.65 & 34.83 & 48.11 & 36.30 & 29.00 & 35.98 & 27.96 & 49.42 & 9.40 \\
\hline & & & 5 & 13.63 & 42.00 & 39.00 & 7.00 & 32.00 & 44.08 & 39.33 & 38.67 & 44.03 & 34.20 & 46.57 & 9.35 \\
\hline & & & 10 & 15.93 & 51.33 & 29.33 & 7.54 & 30.50 & 50.17 & 37.29 & 36.17 & 36.30 & 28.90 & 53.28 & 8.10 \\
\hline & & & 20 & 16.33 & 44.00 & 36.67 & 7.44 & 39.17 & 47.73 & 36.21 & 36.67 & 40.15 & 31.66 & 45.32 & 8.05 \\
\hline & \multirow{16}{*}{3000} & \multirow{4}{*}{0} & 0 & 12.07 & 50.83 & 38.33 & 7.30 & 30.17 & 42.79 & 30.23 & 33.00 & 33.20 & 27.80 & 39.85 & 9.80 \\
\hline & & & 5 & 16.47 & 45.00 & 35.00 & 7.13 & 30.83 & 44.18 & 34.86 & 29.00 & 36.88 & 29.79 & 39.60 & 7.20 \\
\hline & & & 10 & 11.83 & 47.17 & 34.33 & 8.36 & 33.00 & 50.71 & 37.34 & 33.17 & 41.86 & 33.22 & 47.28 & 8.50 \\
\hline & & & 20 & 19.47 & 35.83 & 38.33 & 6.75 & 27.33 & 36.47 & 28.08 & 29.50 & 31.98 & 25.18 & 46.67 & 8.95 \\
\hline & & \multirow{4}{*}{250} & 0 & 17.77 & 40.33 & 38.83 & 7.33 & 30.17 & 44.26 & 32.88 & 36.00 & 40.13 & 29.95 & 48.25 & 8.05 \\
\hline & & & 5 & 16.90 & 45.00 & 35.00 & 7.13 & 30.83 & 48.50 & 34.68 & 29.00 & 40.54 & 31.51 & 50.00 & 9.80 \\
\hline & & & 10 & 14.03 & 41.50 & 35.50 & 6.85 & 27.33 & 36.85 & 27.46 & 25.00 & 35.09 & 28.70 & 47.70 & 8.10 \\
\hline & & & 20 & 19.17 & 35.33 & 42.83 & 6.91 & 35.00 & 38.29 & 27.25 & 31.50 & 35.10 & 28.37 & 46.93 & 8.10 \\
\hline & & \multirow{4}{*}{500} & 0 & 12.73 & 45.33 & 33.67 & 6.59 & 30.67 & 44.21 & 30.12 & 28.67 & 33.79 & 26.27 & 44.98 & 8.50 \\
\hline & & & 5 & 20.27 & 44.50 & 38.00 & 7.58 & 32.67 & 50.90 & 32.25 & 30.50 & 42.46 & 33.62 & 43.82 & 8.95 \\
\hline & & & 10 & 19.70 & 38.33 & 35.00 & 7.21 & 26.83 & 37.69 & 28.59 & 33.50 & 40.39 & 33.70 & 52.27 & 9.80 \\
\hline & & & 20 & 18.23 & 41.67 & 33.33 & 6.15 & 30.33 & 41.14 & 29.32 & 28.67 & 35.56 & 29.83 & 54.80 & 8.90 \\
\hline & & \multirow{4}{*}{1000} & 0 & 14.77 & 39.17 & 32.50 & 7.52 & 30.83 & 41.08 & 29.60 & 33.83 & 37.97 & 32.32 & 49.52 & 7.20 \\
\hline & & & 5 & 18.90 & 37.50 & 45.33 & 6.97 & 40.67 & 46.46 & 36.80 & 31.83 & 40.92 & 34.10 & 57.95 & 7.20 \\
\hline & & & 10 & 15.03 & 45.33 & 42.33 & 7.56 & 33.50 & 47.07 & 35.19 & 39.83 & 39.23 & 31.75 & 41.68 & 8.50 \\
\hline & & & 20 & 11.97 & 34.50 & 37.17 & 6.86 & 30.33 & 38.14 & 29.16 & 34.83 & 35.04 & 29.77 & 44.60 & 7.65 \\
\hline
\end{tabular}


Influence of magnetized water and some fertilizers on the growth of two cultivars of (Pistacia vera L.)

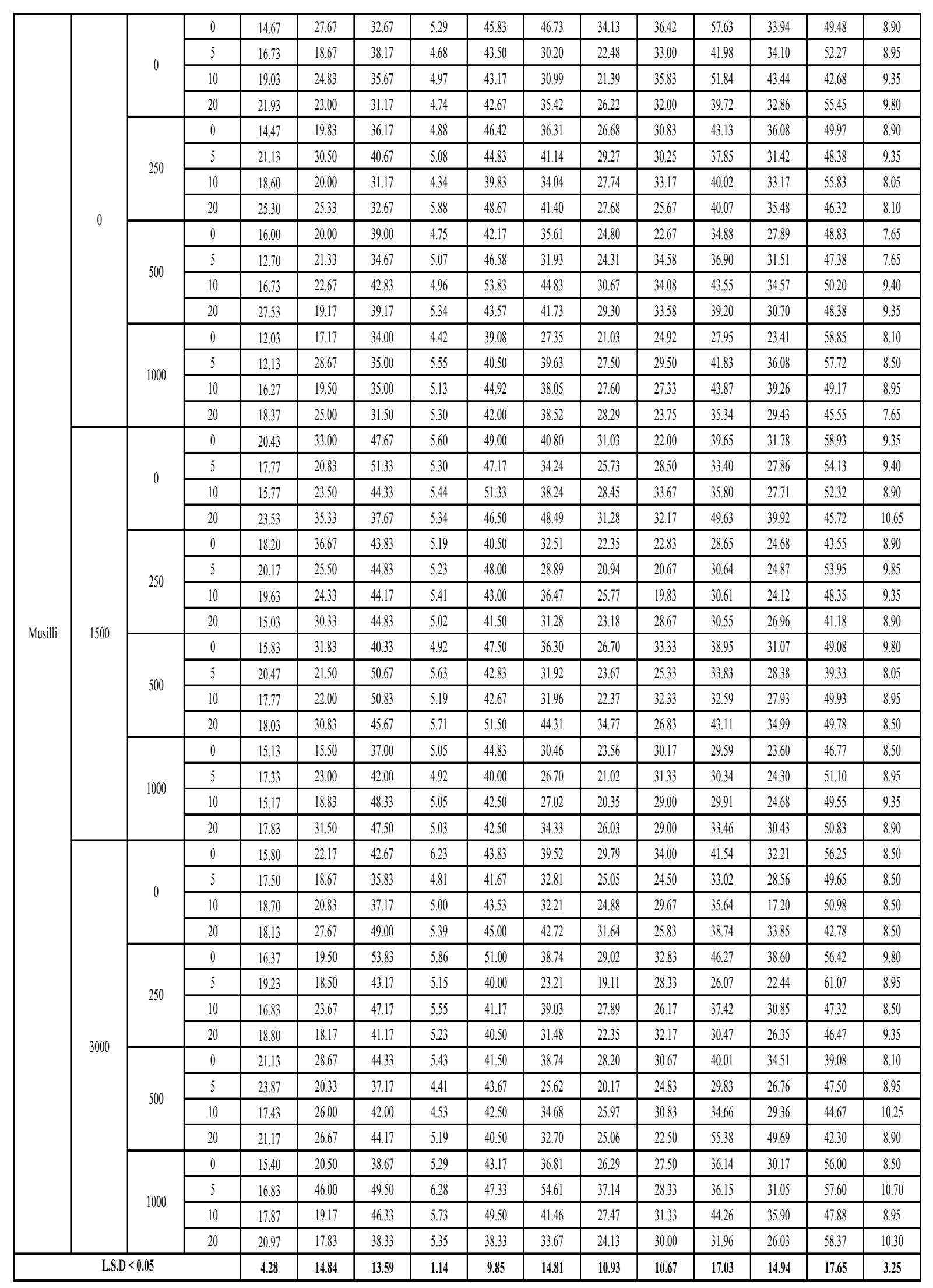




\section{References}

[1]. J. Khan, M. A. Rauf, Z.A. Haroon-ur-Rashid and M. S. Khattak, Different stratification techniques on seed germination of pistachio cv. wild, Pakistan, J. Bio. Sci. 2(4), 1999, 1412-1414.

[2]. J. Crane, C. and B. T. Iwakiri, Morphology and reproduction in pistachio. Hort. Rev.3: 379-393.(C.F. Picch-ioni G. A. (1997). Macronutrient allocation to leaves and fruit of mature alternate-bearing pistachio trees: magnitude and seasonal patterns at the whole-canopy level, Amer. Soc. Hort. Sci. J. 122(2), 1981, 267-274.

[3]. V.Tavallalo, and M. Rahimi, Effect of rootstock on nutrition acquisition by leaf, kernel and quality of pis-tachio (Pistacia vera L.). Amer-Eurasian J. Agr \& Env. Sci. 2(3), 2007,240-246.

[4]. Gh. Davarynajad, T. N. Peter and E. Davarynajad, Concentration of some major elements in the kernel of pistacia atlantica desf., P. vera variety "sarakhs","badami zarand" and cultivated pistachio in Iran, International J.of Nuts and Related Sci. 3(1), 2012, 7-12.

[5]. M. Al- KhazanB. M. Abdullatif and N. Al. Assaf, Effect of magnetically treated water on water status, chlorophyll pigments and some elements content of Jojoba (Simmondsia chinensis L.) at different growth stages, African J. of Env. Sci. and tech. 5(9), 2011, $722-731$.

[6]. M. Afrousheh, M. Ardalan, H. Hokmabadi and M.Afroushi, Visual difficiency and multi deficiency sympt-oms of macro and micro nutrient elements in pistachio seedling (Pistacia vera L.). CIHEAM, 2010, http://am.cheam.org/article.php?IDPDF=801283

[7]. E. Herrera, Growing pistachios in new Mexico, New Mexico state university, 1997.

[8]. H. S. Alti, S. Arpaci and H. Ayanoglu, Comparision of seedling characteristics of some pistacia species, CIHEAM, 2001, http://am.ciheam.org/article.php?IDPDF=1600178:215-218.

[9]. Drs. Patrick, H. Brown, L. Ferguson and G. Picchion, Boron boosts pistachio yields. Fluid Jor. , 1995, 1-3.

[10]. Life sources, Inc, Magnetic water. Life sources, Inc. fair oaks, California, 2003, www.life-sources.com.

[11]. M. J. M. Al-Kaabi, Effect of using magnetic water in irrigation and foliar application of area, Zinc and Iron on growth of mahaly orange, MSc. thesis, college of agriculture, university of Baghdad, Iraq, 2006.

[12]. A. M. S. Abdul-Qados and M. Hozayn, Magnetic water technology, a novel tool to increase growth, yield and chemical constituents of lentil (Lens esculenta) under greenhouse condition, Amer.-Eurasian J. Agr. \& Env. Sci. 7(4), $2010,457-462$.

[13]. M. Hozayn, A. M. S. Abdul-Qados and A. A. Abdel-Monem, Utilization of magnetic water technologies in agriculture: response of growth, some chemical constituents and yield components of some crops for irrigation with magnetized water, International conference of water Resources and Arid Env., Saudi Arabia, 2010, 381- 387.

[14]. E. Tayari and A. R. Jamshidi, Effect of Tillage methods and use magnetic water on green house cucumber yield in north Khuzestan, Iran. Advances in Env. Bio. 5(10), 2011, 3384-3386.

[15]. M. Al-Khazan, B. M. Abdullatif and N. Al-Assaf, Effect of magnetically treated water on water status, chlorophyll pigments and some elements content of jojoba (Simondsia chinensis L.) at different growth stages, African J. of Env. Sci. and technology 5(9), $2011,722-731$.

[16]. O. Sadeghipour and P.Aghaei, Improving the growth of cowpea (vignaunguiculata L. Walp.) by magnetized water, J. of Biodiversity and Env. Sci. 3(1), 2013, 37-43.

[17]. A. F. Demirkiran and M. C. Cengiz, Effect of different organic materials and chemical fertilizers on nut-rition of pistachio (Pistacia vera L.) in organic arboriculture, African J. of Biotechnology 9(38), 2010, 6320-6328.

[18]. SAS, Statistical analysis system Institute, Cary NC, USA, 2005.

[19]. J. Merkwell, C. Osterman and J. L. Mitchel, Calibration in the Minolta SPAD-502 leaf chlorophyll meter, Photosynthesis research 46, 1995, 467-472.

[20]. S. Coste, C. Barolato; C. Leroy; E. Marcon; A. Renaud; A. D. Richardson; J. C. Roggy; H. Schiman; J. Uddling and B. Herault, Assessing foliar chlorophyll contents with the SPAD-502 chlorophyll meter: a calibration test with thirteen tree species of tropical rainforest in French Guiana, Ann. For. Sci. 67(607), (2010), 1-5.

[21]. J. A. Hardim; M. W. Smith; P. R. wecjier and B. S. Cheary, In Situ measurement of pecan leaf nitrogen concentration using a chlorophyll meter and vis-near infrared multispectral camera, HortSci. 47(7), (2012), 955-960.

[22]. M. Kord, L. Derakhshan, H. Memarian and A. Tajabadipour, Effects if high boron concentration on boron uptake and growth of pistachio seedlings, $19^{\text {th }}$ world congress of soil Sci., soil solutions for changing world, Australia, 2010. 